\title{
Free and Bound States of Ions in Ionic Liquids, Conductivity, and Underscreening Paradox
}

\author{
Guang Feng, ${ }^{1,}$ Ming Chen, ${ }^{1}$ Sheng Bi, ${ }^{1}$ Zachary A. H. Goodwin, ${ }^{2,3}$ Eugene B. Postnikov, ${ }^{4}$ \\ Nikolai Brilliantov, ${ }^{5,6, \uparrow}$ Michael Urbakh, ${ }^{7,5}$ and Alexei A. Kornyshev ${ }^{3,8,8}$ \\ ${ }^{1}$ State Key Laboratory of Coal Combustion, School of Energy and Power Engineering, Huazhong \\ University of Science and Technology, Wuhan, \\ Hubei 430074, China \\ ${ }^{2}$ Department of Physics, Imperial College London, South Kensington Campus, \\ London SW7 2AZ, United Kingdom \\ ${ }^{3}$ Department of Chemistry, Imperial College London, Molecular Sciences Research Hub, \\ White City Campus, London W12 OBZ, United Kingdom \\ ${ }^{4}$ Theoretical Physics Department, Kursk State University, Radishcheva Street, 33, Kursk 305000, Russia \\ ${ }_{5}^{5}$ Skolkovo Institute of Science and Technology, Moscow 121205, Russia \\ ${ }^{6}$ Department of Mathematics, University of Leicester, \\ University Road, Leicester LE1 7RH, United Kingdom \\ ${ }^{7}$ School of Chemistry, The Sackler Faculty of Science, Tel Aviv University, \\ Ramat Aviv, Tel Aviv 69978, Israel \\ ${ }^{8}$ Thomas Young Centre for Theory and Simulation of Materials, Imperial College London, \\ South Kensington Campus, London SW7 2AZ, United Kingdom
}

(Received 27 July 2018; revised manuscript received 5 February 2019; published 6 May 2019)

Using molecular dynamics simulations and theoretical analysis of velocity-autocorrelation functions, we study ion transport mechanisms in typical room-temperature ionic liquids. We show that ions may reside in two states: free and bound with an interstate exchange. We investigate quantitatively the exchange process and reveal new qualitative features of this process. To this end, we propose a dynamic criterion for free and bound ions based on the ion trajectory density and demonstrate that this criterion is consistent with a static one based on interionic distances. Analyzing the trajectories of individual cations and anions, we estimate the time that ions spend in bound "clustered states" and when they move quasifreely. Using this method, we evaluate the average portion of "free" ions as approximately $15 \%-25 \%$, increasing with temperature in the range of 300-600 K. The ion diffusion coefficients and conductivities as a function of the temperature calculated from the velocity and electrical-current autocorrelation functions reproduce the reported experimental data very well. The experimental data for the direct-current conductivity (constant ionic current) is in good agreement with theoretical predictions of the Nernst-Einstein equation based on the concentrations and diffusion coefficients of free ions obtained in our simulations. In analogy with electronic semiconductors, we scrutinize an "ionic semiconductor" model for ionic liquids, with valence and conduction "bands" for ions separated by an energy gap. The obtained band gap for the ionic liquid is small, around $26 \mathrm{meV}$, allowing for easy interchange between the two dynamic states. Moreover, we discuss the underscreening paradox in the context of the amount of free charge carriers, showing that the obtained results do not yet approve its simplistic resolution.

DOI: 10.1103/PhysRevX.9.021024

*gfeng@hust.edu.cn

†nb144@leicester.ac.uk

*urbakh@post.tau.ac.il

§a.kornyshev@imperial.ac.uk

Published by the American Physical Society under the terms of the Creative Commons Attribution 4.0 International license. Further distribution of this work must maintain attribution to the author(s) and the published article's title, journal citation, and DOI.

\author{
Subject Areas: Chemical Physics, \\ Computational Physics, \\ Statistical Physics
}

\section{INTRODUCTION AND BACKGROUND}

\section{A. Ionic liquids: Dense, strongly correlated room-temperature plasmas}

The rediscovery of room-temperature ionic liquids (RTILs) was a revolution in chemistry [1]. RTILs, solvent-free electrolytes, are almost universal solvents for synthesis and catalysis [1]. In the past, we could "count" solvents, but for RTILs there are practically an unlimited 
number of them. Easily mixable with each other, "cocktails" of RTILs offer additional variety of "designer solvents" $[2,3]$. As electrolytes, RTILs are interesting for electrocatalysis [4-6]: They sustain higher applied voltages than aqueous electrolytes, with their ions not taking part in electrochemical reactions, which can speed up electrocatalytic reactions of the solutes. For the same reason, together with nonvolatility and endurance of higher temperatures, these electrolytes seem promising in various energy devices, from supercapacitors to fuel cells and batteries [4-6]. For theorists, RTILs are a unique state of matter: dense roomtemperature strongly Coulomb-correlated ionic plasmas.

Since ions in RTILs are packed at liquid density, one expects excluded volume effects to be important. The renowned manifestation of such effects is in the properties of the electrical double layer (EDL) at electrodes: Instead of the standard Gouy-Chapman $U$ shape of capacitancevoltage curves, "bell" and "double-hump-camel" shapes appear [7,8]. The decreasing branches of capacitance emerge due to a restriction on the increase of the local ionic concentration in response to electrode charging: At large electrode polarizations, the thickness of the EDL grows, and the capacitance, which is roughly inversely proportional to it, decreases [9]. At small polarizations, the EDL could first compress if the effective distance to the "center of mass" of the countercharge reduces; this behavior delivers a camelshaped capacitance. A source of such a compression may be filling the natural voids in RTILs [8] or reorientation of charge heads and neutral tails of ions [10]. Estimates, however, show that the volume fraction of voids in RTILs comprises just a few percent $[11,12]$. So, what else can stand behind the countercharge compression? To understand this effect, we need to explore in detail the ion association and dynamics in RTILs.

\section{B. Screening puzzle and ion-clustering concept}

One may have expected that the high ion density in RTILs would result in a short-range screening of an external electric field. However, a few experiments recently seemed to show the opposite [13]. Using surface force apparatus, the Israelachvili group measured forces between a charged mica surface and gold electrode separated by RTILs. A striking result was reported: At large distances between the surfaces, the force seemed to obey the law, valid for two overlapping EDLs, but with an extraordinary long decay length, earlier observed only for very dilute electrolytes. Consequently, a conjecture [14] was raised that pure RTILs are effectively dilute electrolytes, in which most of the ions are bound into neutral clusters (ion pairs or larger aggregates) with only a minute number of ions participating in screening.

Once adopting this picture, the rest is straightforward: (i) Clusters of ions are majorly neutral and have internal polarizability that provides dielectric screening to free ions; (ii) as, hypothetically, only free ions contribute to the formation of EDLs, if their number is small, extra-long screening lengths would emerge. Initially, this conjecture met resistance [15]. But later, Perkin and co-workers obtained similar results, measuring the structural forces between mica surfaces [16]. Gebbie et al. then published a minireview about this phenomenon [17]. Perkin's group put these findings in a broader picture of nonmonotonic dependence of the screening length on the electrolyte concentration [18]. Indeed, in dilute ionic solutions, most ions are free and contribute to screening; thus, the increase of ion concentration makes the Debye length shorter, but with further increase of concentration, more ions get bound into pairs and larger clusters, and thus stop contributing to screening. Such ion pairing or clustering concepts in electrolytes were developed from old to modern times [19-21].

But is it true that most ions in RTILs are clustered? Various estimates $[20,22,23]$ suggested that the expected degree of clustering is not enough to explain the observations by Gebbie et al. [14] in such a simple way. However, before looking for alternative explanations, it is necessary to identify whether we can speak about two states of ions, what is the exchange or balance between them, and how they contribute to conductance of RTILs, as well as to the EDL formation.

The cluster concept in RTILs is actually not new. Hu and Margulis [24] exploited the idea of long-living clusters of ions for the interpretation of the so-called "red-edge effect" (REE) in the fluorescence of the organic probe 2-amino-7nitrofluorene dissolved in 1-butyl-3-methylimidazolium hexafluorophosphate $\left([\mathrm{Bmim}]\left[\mathrm{PF}_{6}\right]\right)$. They focused on establishing the relation between REE and dynamic heterogeneity in RTILs, which is crucial for the data interpretation [25]. Analyzing the van Hove self-diffusion correlation function [32], they found that most ions diffuse much slower than expected from the Gaussian diffusion but some diffuse faster. It was also found that there is a poor correlation between the motions of the two kinds of ions, whereas within each kind the correlations are strong.

Next, in some protic RTILs using electrospray ionization mass spectrometry (ESI-MS), Kennedy and Drummond [33] observed the formation of aggregates of ions, the size of which depends on the nature of the cation and anion. They proposed that RTILs with strong tendency for ion clustering can be classified as "poor ionic liquids," in analogy with weak electrolytes. Generally, ESI-MS has been the principal technique to corroborate the ion cluster model, in which the bulk structure is depicted as a sea of polydisperse aggregates [34,35].

Thus, there is strong experimental evidence in favor of the existence of clustering in RTILs, which supports the two-state model for such liquids, but there is no unified view on the scale of this effect, which may vary from liquid to liquid [36].

\section{Nature of compacity, and overscreening vs underscreening}

Independent of data interpretation, the work by Gebbie et al. had an immediate effect. The interpretation of 
compacity, $\gamma$, defined initially as the ratio of the average concentration of ions to their maximal local concentration, was reconsidered and the mean-field theory of EDL was modified [23,41]. Instead, it was considered as a ratio of the number of free charge carriers to the total number of ions. For given environmental conditions, the bulk value of $\gamma$ can be considered as a set parameter for each RTIL. However, near the interface, across the EDL, the concentration of charge carriers may vary. Indeed, in the inhomogeneous electric field, the balance from the clustered state to the free state of ions shifts in favor of the free state [23].

Recently, Gavish et al. [42] and Rotenberg et al. [43] suggested alternative theoretical explanations of the underscreening effect. Their arguments were based, respectively, on the phenomenological density-functional approach and the mean-spherical approximation. At high electrolyte concentrations, these authors have demonstrated the emergence (at the interface [42] and in the bulk [43]) of self-assembled structures manifested in an extended oscillating internal domain in the spatial ion-ion correlation functions and long monotonous tail beyond the oscillation range. But the tail did not come out as long as seen in the experiments. The emergence of oscillations in charge-density correlation functions was first predicted by Kirkwood [44] and has been reestablished and further explored by other authors within different models and theoretical techniques [45-47]. In a very recent paper, Kjellander [48] has reported a detailed analysis of the emergence of renormalized and oscillating mode screening, discussing the "transient associations of each ion with several ions of opposite charge." He speculates about the possibility of the emergence of very long decay lengths caused by the effect of ion pairing. In another recent paper, Ciach [49] presented a density-functional linear response theory for an

(a)

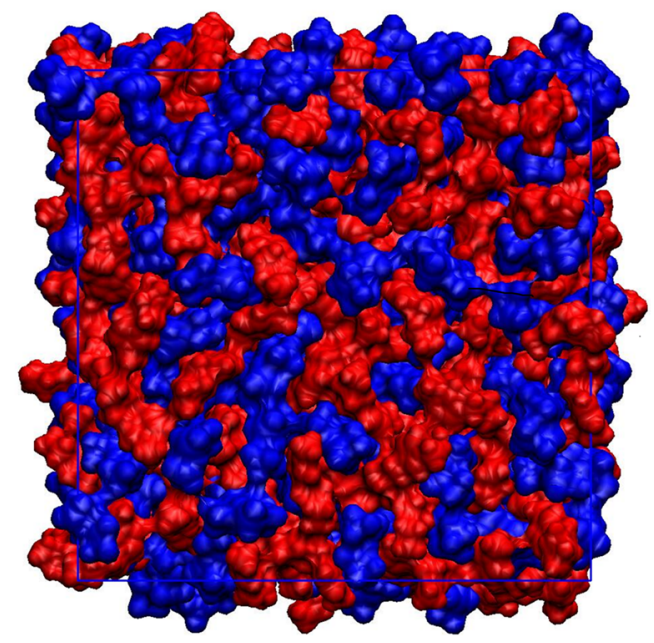

ionic liquid or concentrated ionic solution in a structureless solvent near a charged wall, which shows the emergence of decaying oscillatory profiles of the potential and chargedensity distributions, and the potentiality of an emergence of long decay ranges, which has not been yet explored.

Here we wish to stress that the crossover between oscillating and monotonic patterns of ionic correlation functions can be considered as a manifestation of the clustered and free-ion assemblies (cf. similar ideas of Kjellander [48]). Indeed, clusters imply oscillations of charge density in them and free ions contribute to "tails." Similarly, estimating the portion of ions contained in the former and in the latter could give independent evidence of the degree of clustering in RTILs.

The ion pairing and clustering plays an important role in the conductivity of RTILs, which roughly obeys the NernstEinstein relationship with the measured diffusion coefficient. The deviations from it lead to smaller conductivities attributed to correlations and ion pairing [50]. This interpretation has been debated, with some authors suggesting that the concept of ion pairing and clustering in RTILs is not required [51,52] but some supporting it [53]. There have been investigations into the properties of ion pairs in RTILs [54], but we are not aware of any reports estimating the fraction of ions in a state that contribute to conductivity. This is the gap we aim to bridge in the present study.

\section{Key questions to answer}

The key questions that we attempt to answer in this work are as follows:

(i) Is the idealized picture of two states for ions physically justified and does an unambiguous definition of such states exist?

(b)

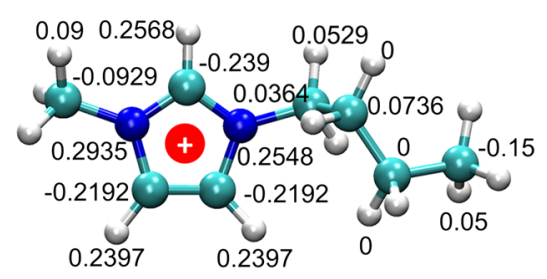

(c)

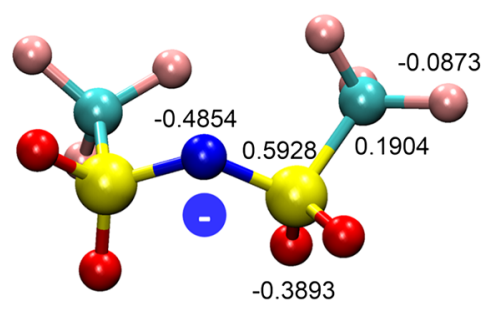

FIG. 1. Molecular dynamics simulation of bulk RTIL [Bmim][TFSI]. (a) Snapshot of MD simulation (red and blue colors indicate cations and anions, respectively). Molecular structures of cation $\mathrm{Bmim}^{+}$(b) and anion $\mathrm{TFSI}^{-}$(c). The numbers near atoms denote the partial charges on each atom in the all-atom model of ions used for MD modeling (unit: elementary charge). The solid red circle with the sign "+" represents the cation center (i.e., the mass center of the ring), and the solid blue circle with the sign "--" indicates anion center (i.e., the mass center of the whole anion). 
(ii) What is the fraction of "free" and "bound" ions, and what is their role in electric conductance and screening?

(iii) How can the interstate exchange kinetics be quantified, and what is the energy difference ("band gap") between the free and bound states?

In order to test the validity of the two-state concept, we address these questions as well as a few accompanied ones related to the mechanisms of ionic diffusion and conductance in RTILs. In so doing, we exploit MD simulations which allow us to trace the trajectories of individual ions, as well as to obtain the statistical characteristics of their motions such as self-diffusion coefficients, conductivities, and the like, and to investigate their temperature dependence. As a typical example of a RTIL, 1-n-butyl-3-methylimidazolium bis(trifluoromethanesulfonyl)imide ([Bmim] [TFSI]) is chosen for study in the first place, but two other RTILs are also considered for comparison to test the universality of the conclusions. We use detailed molecular models for these liquids (for [Bmim][TFSI], see Fig. 1), which help make a direct comparison of the simulation results with the experimental data. We perform MD simulations of bulk RTILs at different temperatures and extract all necessary information to quantify the ionic transport and interstates exchange kinetics. Such study allows us to evaluate the viability of the two-state concept. Using a set of somewhat complementary methods, we not only validate this concept but also reveal new qualitative features of the two-state dynamics. Namely, we demonstrate (i) the nonPoissonian nature of the interstate exchange process and (ii) "dynamic decoupling" for the in-state molecular motion - the statistical independence of time-correlation functions in each state (more details are given below). The results for RTIL [Bmim][TFSI] are presented in the main text, and similar results for another two RTILs are shown in the Supplemental Material [108]. We also test the sensitivity of results to the size of the system. The details of the computational and analytical methods are presented in Appendixes A-D.

\section{RESULTS AND DISCUSSION}

Generally, there may be two alternative definitions of the free and bound states - a kinetic one and a static one. The former definition refers to the ability of ions to participate in electrical direct-current (dc) conductivity of RTILs. The latter definition is associated with the equilibrium properties of the liquid, such as the distance between the positive and negative ions in a pair or cluster.

\section{A. Kinetic criterion: Trajectory-density analysis}

First, we analyze the motion of individual cations and anions in the RTILs' crowded environment, which will provide important information on the structure and dynamics of the liquid [55]. In Figs. 2(a) and 2(b), respectively, the trajectory of the center of one cation and anion, which is arbitrarily chosen, is displayed. It can be seen that an ion moves for a while reentrantly in anticorrelated fashion in a confined volume and then "speeds up," undergoing correlated persistent motion outside that volume to later get trapped in a new domain. This motion is seen from denser trajectory clouds connected by sparse ones. The boxcounting method [56] is adopted to obtain the local trajectory density (LTD). Specifically, we first focus on one selected ion (cation or anion) and get all of its trajectory points [Figs. 2(a) and 2(b)]. Then, we compute the LTD via the box-counting method [Figs. 2(c) and 2(d)], dividing the simulation cell into elementary cubicles with a size of $0.3 \mathrm{~nm}$ (in Appendix B, we provide a justification of this method and show the negligible effect of the trajectory length on LTD). To differentiate the ion states, we conventionally
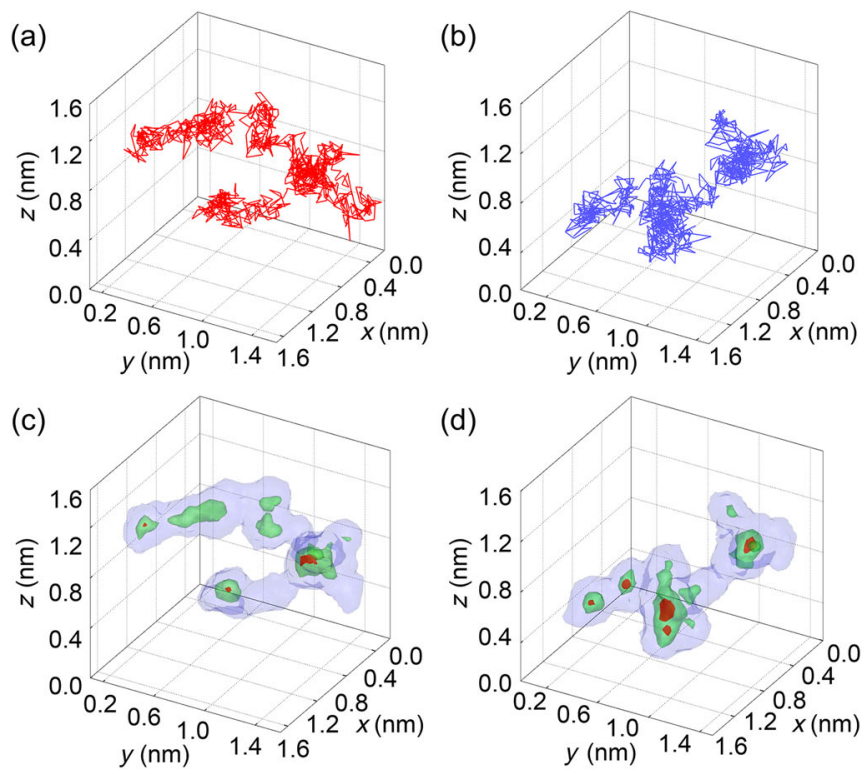

(e)

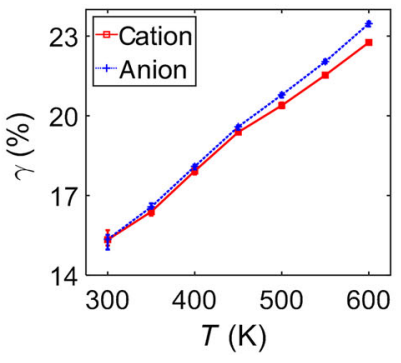

(f)

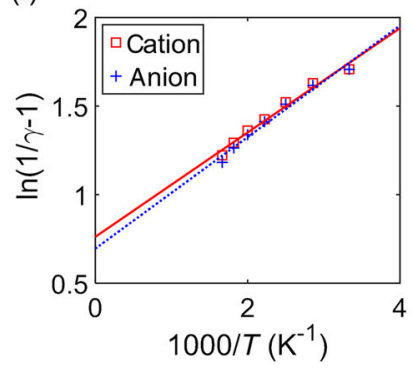

FIG. 2. Two states of ion dynamics revealed by ion trajectories based on MD simulations of [Bmim][TFSI]. Trajectories of arbitrarily chosen cation (a) and anion (b) and the contour map of trajectory density of the cation (c) and anion (d). (e) The percentage of free ion ( $\gamma$, given in percentage) as a function of the temperature (lines are guides for the eye). (f) Plot for the percentage of free ions based on Eq. (1) (lines represent the linear fit). Gray, green, and red colors in (c),(d) represent small, middle, and high densities. 
assume an ion to be in a "free state" when its LTD is smaller than the average density of all trajectories, otherwise, it is considered to be in a bound state.

The percentage of free ions $\gamma$ is computed as a fraction of time spent by each cation or anion in thus-defined free state, which we then average separately over all cations and all anions. As one can see from Fig. 2(e), the free-ion percentage increases with the temperature. Moreover, these values for cations and anions are very close to each other, with minor differences only at $T>450 \mathrm{~K}$ [57]. Considering the portions of free cations and anions to be roughly the same, these portions can be described by the following equation (see Appendix C):

$$
\gamma=\frac{n}{n_{\mathrm{tot}} / 2}=\frac{1}{1+\frac{1}{2} \zeta e^{\frac{E_{g}}{k_{B} T} \frac{\Delta S_{\mathrm{ex}}}{k_{B}}}},
$$

where $n$ is the number density of free cations or anions ( $\left.n=n_{+} \approx n_{-}\right)$, and $n_{\text {tot }}$ is the total number density of ions including free ions and bound ions, $E_{g}$ is the energy gap between the free and bound states, $\Delta S_{\mathrm{ex}}$ is the corresponding change of the excess entropy, and $k_{B}$ and $T$ are, respectively, the Boltzmann constant and temperature. Equation (1) with the constant $\zeta=1$ has been derived in Ref. [41] using some simplifying assumptions. Generally, $\zeta$ depends on the properties of the ions and neutral clusters in a RTIL; a more detailed explanation of the meaning of Eq. (1) along with the derivation generalizing the approach of Ref. [41] is given in Appendix C.

Now we rewrite Eq. (1) as $\ln [(1 / \gamma)-1]=\{\ln \zeta-$ $\left.\left[\ln 2+\left(\Delta S_{\mathrm{ex}} / k_{B}\right)\right]\right\}+\left(E_{g} / k_{B} T\right)$ and plot the simulated values of $\ln [(1 / \gamma)-1]$ vs $(1 / T)$. Since the (possible) dependence on the temperature of $\ln \zeta$ is logarithmically weak, it may be treated with good accuracy as a constant for the addressed temperature interval. Therefore, the gap energy can be estimated from the slope of the dependence $\ln [(1 / \gamma)-1]$ vs $(1 / T)$, which is shown in Fig. 2(f), giving $E_{g}=2.5 \mathrm{~kJ} \mathrm{~mol}^{-1}(0.026 \mathrm{eV})$ for cations and $E_{g}=$ $2.6 \mathrm{~kJ} \mathrm{~mol}^{-1}(0.027 \mathrm{eV})$ for anions; that is, these quantities are equal within the accuracy of the measurements. To estimate the entropy change $\Delta S_{\text {ex }}$, one needs some additional assumptions (see more details in Appendix C).

\section{B. Static criterion: Structural analysis of ion pairing}

The above concepts of free and bound state ions rise from the analysis of temporal trajectory by tracing each ion. That approach does not specify how the ions are associated with each other. We may try another approach based on the conventional definition of ion pairing. Considering that in RTILs neighboring cations and anions strongly attract each other, the association among them could be described in terms of ion pairing [54,58]. This concept has been clearly justified in electrolytic solutions, but in dense ionic systems, such as RTILs, it is hard to distinguish "who is paired with whom" and whether pairs are not just parts of bigger clusters. Nevertheless, let us define the cation and anion as an ion pair when their ion centers are within a certain distance from each other, taking such a distance to be the sum of the radii of the oppositely charged ions. Using the free-volume method [59], the free volume of the anion and the effective free volume of the cation are computed. We call the latter "effective" because we represent the cation only by its ring with two associated methyl groups that together contain most of the charge distribution. Representing ions as spheres [59], their effective radii can be found: $0.286 \mathrm{~nm}$ for the cation and $0.326 \mathrm{~nm}$ for the anion in line with the literature $[60,61]$. It should not be surprising that the effective cation radius appears smaller than the anion's radius; as long as it is used for the purpose of estimation of the distance of the closest approach of the cation and anion in a pair, it makes sense.

Each ion can form only one ion pair with a counterion, the two behaving as a quasisolvent molecule if the cationanion distance is no more than $0.612 \mathrm{~nm}$; ions that are not involved in ion pairing are considered free. With this approach, the percentage of free ions is computed. The results show similar trends to those quantified via trajectory analysis (see the Supplemental Material [108], Fig. S1).

In brief, the criteria based on trajectory density and ion pairing analyses arrive at the same conclusions; i.e., the free ions are the minority (within about $15 \%-25 \%$ ) of all ions in RTILs, their percentage increasing with the temperature.

We like to note that while we use the free-volume method to find the ion radii, we cannot apply another widely used method of hydrodynamic radius, since the latter would depend on such effects as dielectric friction, electrostriction, and electroviscosity [62,63], and the theory that accounts for all these effects in RTILs is presently lacking.

\section{Ion kinetics in RTILs in terms of the two-state model}

Considering the conclusions made in the previous two subsections as well as evidence that ions may successively reside in two states - free and bound - with rather different kinetics, we need to adopt an appropriate method to describe such systems. In the literature, they are called "systems with chemical exchange" [64-66]. Prominent examples are aqueous solutions of proteins where water molecules or magnetic active ions can be in two dynamical states - in a free state of the bulk of the solvent and in a bound state adsorbed on a protein surface $[67,68]$. The overall molecular kinetics in systems with interstate exchange is determined by the molecular motion in the free or bound state and the kinetics of the exchange process. The former is characterized by time-correlation functions, which describe the individual kinetics of a particle averaged over an appropriate ensemble. The latter is characterized by the so-called survival probability functions [53,69-71], 


$$
c(t)=\frac{\langle h(0) h(t)\rangle}{\langle h(0) h(0)\rangle}
$$

where $h(t)$ is the population variable defined as unity if the ion continuously remains in the same state during the time duration $t$, and zero otherwise. Based on the trajectories used for analyzing the free-ion percentage, the survival probability functions are computed and compared with the exponential and biexponential fittings in Fig. 3(a) and in Fig. S2 of the Supplemental Material [108].

If it follows the Poissonian exchange process, the survival probability function should be exponential [72], that is, $c(t)=e^{-t / \tau}$ (here, $\tau$ is the mean residence time), as shown by blue dashed lines in Fig. 3(a) and in Fig. S2 of the Supplemental Material [108], respectively, for cations and anions. The distinct deviation of MD-obtained $c(t)$ from the exponential relaxation indicates the presence of memory effects in the exchange process [73]. Therefore, the exchange processes between two states are not Poissonian and memory effects seem to be important. This is an interesting result, since in previous studies of the exchange kinetics in other systems, such processes have been assumed Poissonian, and moreover, their Poissonian nature has not even been questioned [64-66]. Herein, we analyze the kinetics of the system with non-Poissonian exchange process, including memory effects described by the memory function [74] (see Appendix D for details). Specifically, the observed survival probability function would be described by the biexponential function $c(t)=a e^{-t / \tau_{f}}+b e^{-t / \tau_{s}}$, where $a+b=1$ and $\tau_{f}$ and $\tau_{s}$ denote, respectively, the relaxation times for the fast and slow parts of the process. Shown as green dotted lines in Fig. 3(a) and in Fig. S2 of the Supplemental Material [108], the biexponential functions are found to fit quite well the survival probability functions calculated from MD simulations. Note that the above biexponential dependence is obtained by a direct fitting to the simulation data, which alone cannot rationalize the exact nature of the processes behind the two relaxation times.

Another important function describing the exchange kinetics is $g(t)=-d c(t) / d t$ so that $g(t) d t$ gives the probability for an ion to remain in an initial state (the state at $t=0$ ) up to the time $t$ and pass to another state within the time interval $d t$. The mean residence time then reads

$$
\tau=\int_{0}^{\infty} t g(t) d t=\int_{0}^{\infty} c(t) d t=\tilde{c}(0),
$$

where $\tilde{c}(0)=\tilde{c}(s=0)$ is the according Laplace transform of $c(t)$ (see Appendix D for details).

The mean residence times computed by Eq. (3) decrease with increasing temperature and are close for cations and anions [Fig. 3(b)]. The latter has important physical consequences. It means that ions leave the bound states in cation-anion pairs, and the remaining clusters stay neutral. Those will not contribute to direct-current conductivity. Minor disparity in dissociation of clusters will not be seen in conductivity, because if even charged, their number will be very small, as well as they will diffuse much slower than the individual free ions. (a)

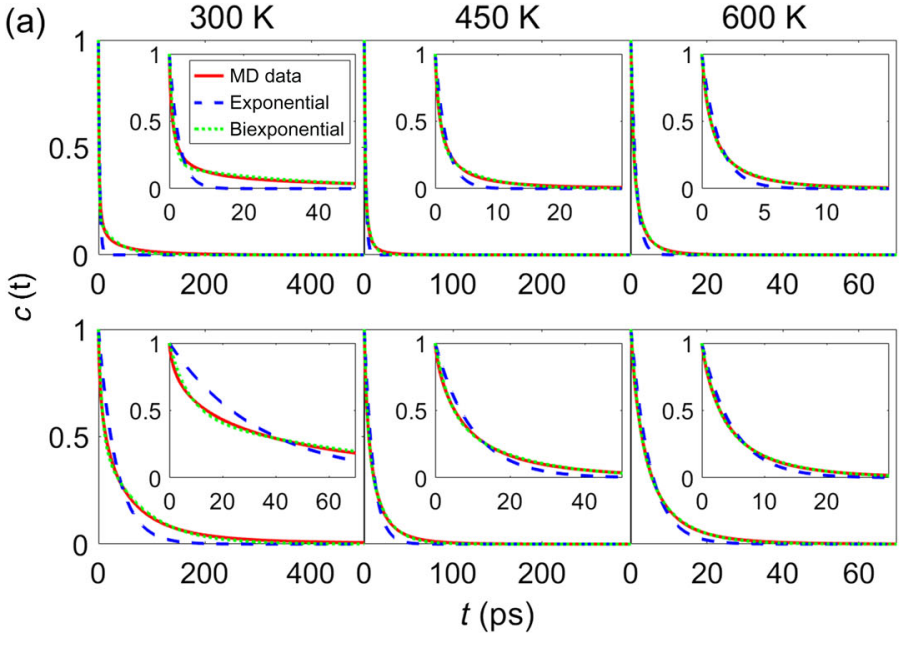

(b)
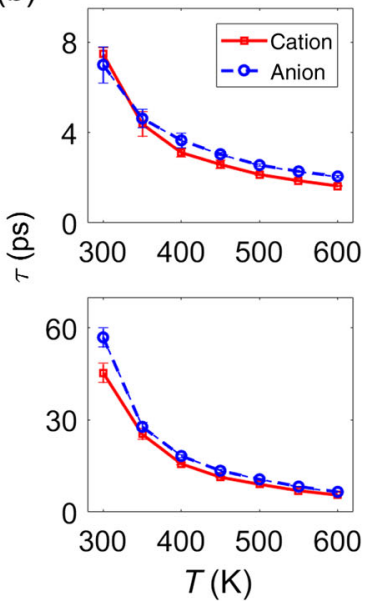

FIG. 3. The kinetics of exchange process for ions in [Bmim][TFSI]. (a) The survival probability functions of cations in free (top panel) and bound (bottom panel) states. Red solid lines are obtained from MD trajectories by Eq. (2); blue dashed and green dotted lines represent the exponential and biexponential fittings, respectively, and biexponential rather than exponential fitting describes $c(t)$ reasonably well. The exponential function is $c(t)=e^{-t / \tau}$, where $\tau$ is the mean residence time; the biexponential function is $c(t)=a e^{-t / \tau_{f}}+b e^{-t / \tau_{s}}$, where $a+b=1$ and $\tau_{f}$ and $\tau_{s}$ denote, respectively, the fast and slow relaxation times (see Table S1 in the Supplemental Material [108] for details). (b) The mean residence time of cations and anions in free (top panel) and bound (bottom panel) states calculated by Eq. (3). 
To quantify the kinetics of ions in the RTIL, we apply now the general theory of time-correlation functions and kinetic coefficients for the systems with interstate exchange. First, we consider the velocity autocorrelation function (VACF),

$$
K_{v}(t)=\frac{\langle\vec{v}(0) \cdot \vec{v}(t)\rangle}{\langle\vec{v}(0) \cdot \vec{v}(0)\rangle},
$$

where $\vec{v}(0)$ and $\vec{v}(t)$ are the ion velocities at the initial instant and time $t$, respectively, and then the diffusion coefficient is obtained as

$$
D=\frac{k_{B} T}{m} \int_{0}^{\infty} K_{v}(t) d t,
$$

where $m$ is the mass of an ion. The averaging in Eq. (4) is performed over an equilibrium ensemble. In equilibrium, with the exchange between two states (free and bound), dynamical balance is held.

As the average times spent by an ion in free and bound states (i.e., the mean residence times) are $\tau_{1}$ and $\tau_{2}$, respectively, the probabilities $p_{i}(i=1,2)$ of belonging to either the free (1) or bound (2) state are related to the residence times as $p_{i}=\tau_{i} /\left(\tau_{1}+\tau_{2}\right)$. Hence, the free-ion percentage reads

$$
\gamma=\frac{\tau_{1}}{\left(\tau_{1}+\tau_{2}\right)},
$$

which is a natural kinetic definition of $\gamma$. Moreover, using MD-obtained data for $\tau_{1}$ and $\tau_{2}$ shown in Fig. 3(b), we can evaluate $\gamma$ via Eq. (6) and get values very close to those computed by means of the trajectory-density method. The overall comparisons between the trajectory-density method, ion-pairing method, and the kinetic definition of $\gamma$ can be found in Fig. S1 of the Supplemental Material [108].

If the dynamics in both states does not depend on each other (although the dynamics in either state may be complicated and non-Markovian), the total VACF $K_{v}(t)$ may be expressed in terms of partial VACFs for free and bound states $K_{v}^{(1)}(t)$ and $K_{v}^{(2)}(t)$ and the functions $c(t)$ and $g(t)$. Simpler expressions may be derived, however, not for the functions themselves but for their Laplace transforms $\tilde{K}_{v}$ and $\tilde{K}_{v}^{(i)}(s)$ (see Appendix D for details):

$\tilde{K}_{v}(s)=\frac{\left(\tilde{G}_{1}+s \tilde{C}_{1}\right)\left(p_{1}+p_{2} \tilde{g}_{2}\right)+\left(\tilde{G}_{2}+s \tilde{C}_{2}\right)\left(p_{2}+p_{1} \tilde{g}_{1}\right)}{\tilde{c}_{1}\left(p_{1}+p_{2} \tilde{g}_{2}\right)+\tilde{c}_{2}\left(p_{2}+p_{1} \tilde{g}_{1}\right)}$,

where the relations between $p_{i}$ and $\tau_{i}$ are given above, $\tilde{c}_{i}=$ $\tilde{c}_{i}(s)$ and $\tilde{g}_{i}=\tilde{g}_{i}(s)=1-s \tilde{c}_{i}(s)$ are, respectively, the Laplace transforms of the functions $c_{i}(t)$ and $g_{i}(t)$, while $\tilde{C}_{i}=\tilde{C}_{i}(s)$ and $\tilde{G}_{i}=\tilde{G}_{i}(s)$ are the Laplace transforms of the products $\tilde{C}_{i}(s)=\int_{0}^{\infty} c_{i}(t) e^{-s t} d t \int_{0}^{t}(t-\tau) K_{v}^{(i)}(\tau) d \tau$ and
$\tilde{G}_{i}(s)=\int_{0}^{\infty} g_{i}(t) e^{-s t} d t \int_{0}^{t}(t-\tau) K_{v}^{(i)}(\tau) d \tau$. Based on Eqs. (5) and (7), the diffusion coefficient can be expressed as

$$
D=\frac{k_{B} T}{m} \frac{\left[\tilde{G}_{1}(0)+\tilde{G}_{2}(0)\right]}{\tau_{1}+\tau_{2}},
$$

where $\tilde{G}_{i}(0)=\int_{0}^{\infty} c_{i}(t) d t \int_{0}^{t} K_{v}^{(i)}(\tau) d \tau$.

For the Poissonian process with the exponential survival function $c_{i}(t)=e^{-t / \tau_{i}}$, where $\tau_{i}$ are the relaxation times for ions in the free $(i=1)$ and bound $(i=2)$ states, the above expressions reduce to formulas available in the literature [75] (see Appendix D for details). For the observed biexponential survival functions $c_{i}(t)=a_{i} e^{-t / \tau_{f, i}}+$ $b_{i} e^{-t / \tau_{s, i}}$, where $a_{i}+b_{i}=1$, and $\tau_{f, i}$ and $\tau_{s, i}$ denote, respectively, the fast and slow relaxation times for the free and bound states, the above functions read

$$
\begin{aligned}
& \tilde{c}_{i}(s)=a_{i}\left(s+\tau_{f, i}^{-1}\right)^{-1}+b_{i}\left(s+\tau_{s, i}^{-1}\right)^{-1}, \\
& \tilde{g}_{i}(s)=a_{i} \tau_{f, i}^{-1}\left(s+\tau_{f, i}^{-1}\right)^{-1}+b_{i} \tau_{s, i}^{-1}\left(s+\tau_{s, i}^{-1}\right)^{-1}, \\
& \tilde{C}_{i}(s)=a_{i} \frac{\tilde{K}_{v}^{(i)}\left(s+\tau_{f, i}^{-1}\right)}{\left(s+\tau_{f, i}^{-1}\right)^{2}}+b_{i} \frac{\tilde{K}_{v}^{(i)}\left(s+\tau_{s, i}^{-1}\right)}{\left(s+\tau_{s, i}^{-1}\right)^{2}}, \\
& \tilde{G}_{i}(s)=a_{i} \frac{\tilde{K}_{v}^{(i)}\left(s+\tau_{f, i}^{-1}\right)}{\tau_{f, i}\left(s+\tau_{f, i}^{-1}\right)^{2}}+b_{i} \frac{\tilde{K}_{v}^{(i)}\left(s+\tau_{s, i}^{-1}\right)}{\tau_{s, i}\left(s+\tau_{s, i}^{-1}\right)^{2}}
\end{aligned}
$$

with the respective mean residence times $\tau_{i}=a_{i} \tau_{f, i}+$ $b_{i} \tau_{s, i}$.

\section{Velocity autocorrelation functions and diffusion coefficients}

To characterize the ion dynamics in the free and bound states, the VACFs and diffusion coefficients are computed for each state and compared with those based on the whole trajectory analysis.

Indeed, the diffusion coefficients obtained from the VACF as well as from the mean square displacement (MSD) have statistical nature (as they explicitly contain an ensemble average in their definitions) and are defined only for an ensemble. The same is true for the exchange process. The mean residence time $\tau_{i}$ quantifies the probability of ions to remain in one state. The trajectory points for an ion in either state (free or bound) include fragments, where an ion resides in a certain state during time $t$ significantly longer than the mean residence time, $t \gg \tau_{i}$ (see Fig. 3). The portion of such fragments decreases with $t$ growing. Hence, conceptually, there is no problem to measure the diffusion coefficient and VACF for either state just using such long intervals of trajectories, where an ion resides in the same state as long as needed for the measurements. To obtain good statistics, an averaging of the results over many runs is a must. 
We illustrate this approach by a representative example considering the cation or anion diffusion in the bound state for $T=300 \mathrm{~K}$. The mean residence time for this state is about 50 ps [see the bottom of Fig. 3(b)]. To obtain an accurate diffusion coefficient, one needs to integrate the VACF over about 100 ps [see Fig. S3(a) in the Supplemental Material [108]]. As it follows from the survival probability which quantifies the fraction of ions that do not change their state during the time interval $t$, the fraction of fragments with length exceeding $t=100 \mathrm{ps}$ is about 12\% [Fig. 3(a)]. This is sufficient for accurate calculations of the diffusion coefficient in the bound state. For other cases, the corresponding fraction of ions may be larger or smaller, but it is still large enough to perform accurate calculations for both the VACFs and diffusion coefficients.

In order to compute the diffusion coefficients in the free or bound state, we first compute the VACFs separately for ions staying all the time in the free and bound states. Each ion trajectory contains shorter and longer fragments that can be attributed to one of the two states [defined in the survival probability function by Eq. (2)], some of which may be as short as 8-ps intervals, whereas others are as long as 120-ps intervals. The statistics of these states are quantified by the survival probability function [Fig. 3(a) and Fig. S2 in the Supplemental Material [108] ]. When we average the VACFs over all such events, the short-living ones may not contribute to the VACF values at longer times. Roughly speaking, almost all ions in the free state at $t=0$ contribute to the initial part of the VACF of free ions. At longer time, fewer ions remain in the free state and contribute to the VACF. In all our simulations of the VACF for free or bound ions, however, we always have enough free or bound ions to describe the asymptotic decay of the VACF to zero.

The VACF profiles in Fig. 4(a) show damped oscillations less pronounced as the temperature increases. But for cations [Fig. 4(a), top panel], the VACFs exhibit an intermediate negative asymptotic plateau, which may be attributed to backscattering due to cation oscillations within an ion "cage" built by other ions in the bound state. With the temperature increasing, the backscattering is weaker, probably due to easier escape of the cations from the cage. Similar trends hold for the VACFs of the free and bound ions, but the latter ion exhibits, as expected, more pronounced oscillatory motions.

The diffusion coefficients of ions in different dynamic states are given by Eq. (5). Using the method proposed in Ref. [77], technically, the diffusion coefficient is first calculated by integrating the VACF over the time as in Eq. (5), and then the diffusion coefficients of ions in different dynamic states are obtained by averaging the "plateau" of the diffusion-time curves (see Fig. S3 in the Supplemental Material [108]). The results are shown in
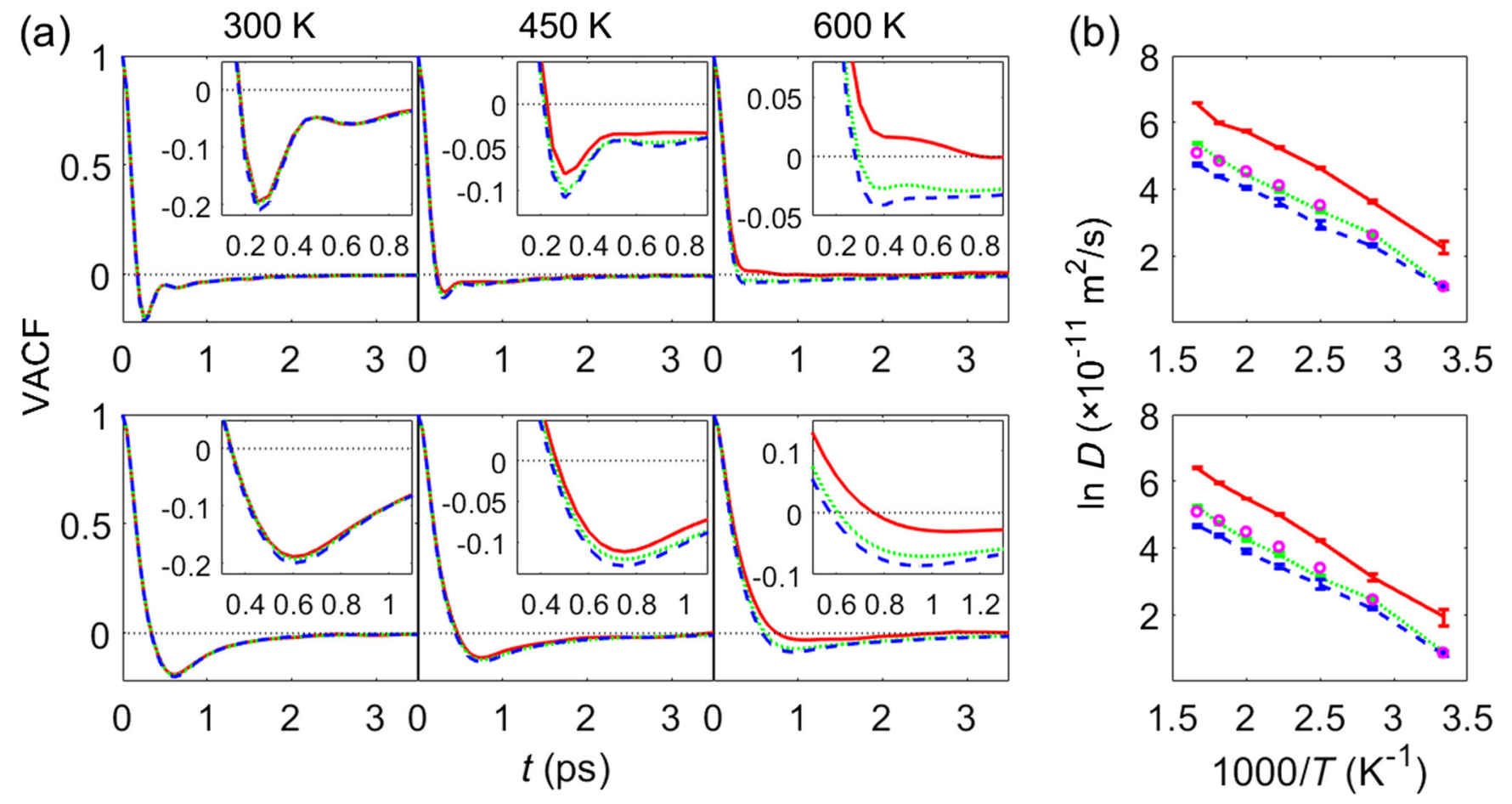

FIG. 4. Impact of the temperature on the ion kinetics in RTIL [Bmim][TFSI]. (a) VACFs of cations (top panel) and anions (bottom panel) at indicated temperatures. (b) Diffusion coefficients of cations (top panel) and anions (bottom panel) as a function of the temperature shown in Arrhenius coordinates. Red solid, green dotted, and blue dashed lines represent free ions, overall ions, and bound ions, respectively. Magenta circles in (b) represent experimental data from Ref. [76]. 
TABLE I. Arrhenius equation fitting of diffusion coefficients with different states.

\begin{tabular}{lcccccccc}
\hline \hline & \multicolumn{2}{c}{ Free state } & & \multicolumn{2}{c}{ Overall state } & & \multicolumn{2}{c}{ Bound state } \\
\cline { 2 - 3 } \cline { 8 - 9 } Ion & $D_{0}$ & $E_{a}$ & & $D_{0}$ & $E_{a}$ & & $D_{0}$ & $E_{a}$ \\
\hline Cation & 4.57 & 20.79 & & 1.07 & 19.77 & & 0.43 & 18.00 \\
Anion & 5.27 & 22.29 & & 1.07 & 20.54 & & 0.47 & 18.67 \\
\hline \hline
\end{tabular}

Note: The term "overall" means that the diffusion coefficients are calculated using entire ion trajectories, and $D_{0}$ is a fitting constant physically corresponding to the hypothetical diffusion coefficient at infinitely high temperature. Unit of $D_{0}: 10^{-7} \mathrm{~m}^{2} / \mathrm{s}$. Unit of $E_{a}: \mathrm{kJ} / \mathrm{mol}$.

Fig. 4(b) - top panel for cations and bottom panel for anions. We find that the overall diffusion coefficients of ions calculated using the entire ion trajectories agree very well with experimental data [76]; ions in the free state diffuse much faster than in the bound state, and with increasing temperature, such a deviation becomes more distinct [Fig. 4(b)], which is compatible with a quasielastic neutron-scattering study [78]. The values of the overall diffusion coefficients calculated through the simulated VACFs are very close as those we obtain based on MSD [79] (shown in Fig. S4 of the Supplemental Material [108]); similar to Ref. [77], we check that the size of the simulation system that we use is large enough to have a negligible effect on those values (see Fig. 8 and discussion in Appendix A).

The significant enhancement of the diffusion of the free ions as compared to the bound ions is accompanied by the backscattering that is particularly pronounced at lower temperatures as seen in their VACFs [Fig. 4(a)]. The enhanced free-ion diffusion may be attributed to more salient motions of ions through virtual voids or channels [80].

Diffusion coefficients as a function of the temperature in Fig. 4(b) are fitted to Arrhenius equation $D=$ $D_{0} \exp \left(-E_{a} / k_{B} T\right)$ resulting in an activation energy $E_{a}$ of approximately $21 \mathrm{~kJ} \mathrm{~mol}^{-1}(0.22 \mathrm{eV})$ for free ions, larger than that for bound ions of approximately $18 \mathrm{~kJ} \mathrm{~mol}^{-1}$ $(0.18 \mathrm{eV})$ (see Table I). This difference can be understood considering that individual ions in a clustered state oscillate in a cage, and each elementary step of their motion requires less reorganization of the environment, resulting in lower activation energy as compared to the motion of free ions. But the motions of ions in a clustered state are anticorrelated, indicating that the presence of confining potential makes diffusion less effective: It makes the preexponential factor much smaller, which wins over the minor decrease of the activation energy.

To compare the simulation results with the theoretical expression in Eq. (7), we adopt the frequency-dependent VACF, i.e., its Fourier component [expressed through the Laplace transform as $\left.\hat{K}_{v}(\omega)=2 \operatorname{Re} \tilde{K}_{v}(s=j \omega), j=\sqrt{-1}\right]$. Calculations of $\hat{K}_{v}(\omega)$ prescribed by Eq. (7) require $\hat{K}_{v}^{(1)}(\omega)$ and $\hat{K}_{v}^{(2)}(\omega)$ obtained separately from the simulations of $K_{v}^{(1)}(t)$ and $K_{v}^{(2)}(t)$ by collecting the trajectories of the two states as well as one parameter of ion percentage [i.e., $p_{1}$ and $p_{2}=1-p_{1}$; see Fig. 2(e)] and three
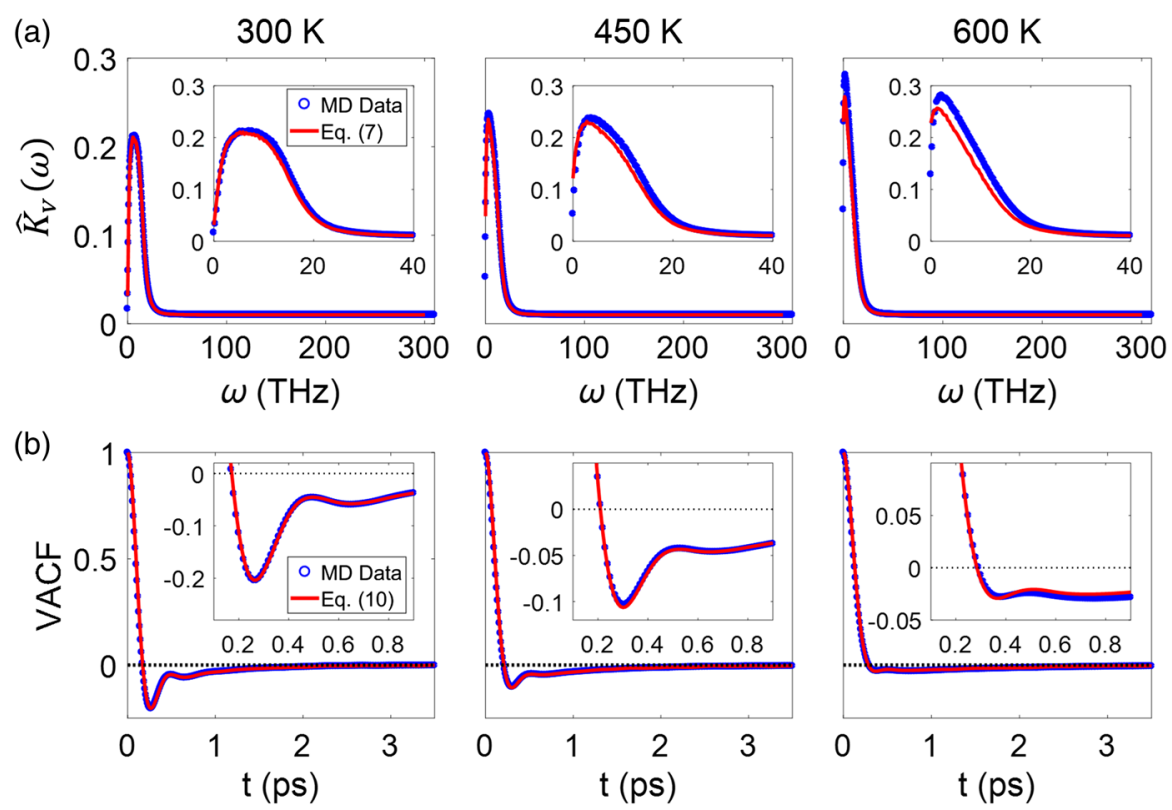

FIG. 5. Two-state theory of VACFs of cations in RTIL [Bmim][TFSI]. (a) Comparison of the Fourier transforms $\hat{K}_{v}(\omega)$ of VACFs obtained in MD simulations and that for the two-state model based on Eq. (7), where $\hat{K}_{v}(\omega)=2 \operatorname{Re} \tilde{K}_{v}(s=j \omega)$. (b) VACFs in the time domain for cations as a linear combination of those of free and bound cations based on Eq. (10) as obtained for the same data as in (a). The statistical coefficient of determination is $0.994,0.979$, and 0.971 for 300,450 , and $600 \mathrm{~K}$, respectively. 
parameters for the free and bound states [i.e., $a_{i}, \tau_{f, i}$, and $\tau_{s, i}, i=1,2$; see Fig. 3(b)] entering Eqs. (7) and (9) using the trajectory analysis (see Appendix D for more details). Shown in Fig. 5(a), the spectra of the function $\hat{K}_{v}(\omega)$ based on Eq. (7) are very close to the ones calculated directly from MD simulations.

We now introduce the notion of correlation times $[74,75,84,85]$. A correlation time of dynamical variable quantifies the time interval during which self-correlations of this variable die out. It is defined through the time integral of its correlation function [see Eq. (D6)]. For instance, the velocity-correlation time $\tau_{v}^{(i)}$ is related to the diffusion coefficient as $\tau_{v}^{(i)}=m D_{i} / k_{B} T=\int_{0}^{\infty} K_{v}^{(i)}(t) d t$ [see Eq. (5)]. From the physical meaning of the velocity-correlation time, it follows that for time $t \gg \tau_{v}^{(i)}$, the velocity-correlation function is negligibly small, $K_{v}^{(i)}(t) \ll$ 1. As it comes out from our simulations, the VACFs die out faster than the transitions between the states take place, and the analysis of MD simulation in this work gives that $\tau_{v}^{(1)} \ll \tau_{1}$ and $\tau_{v}^{(2)} \ll \tau_{2}$.

Under these conditions, Eq. (7) significantly simplifies, yielding (Appendix D)

$$
K_{v}(t)=p_{1} K_{v}^{(1)}(t)+p_{2} K_{v}^{(2)}(t) .
$$

Equation (8) for the diffusion coefficient is simplified according to $D=p_{1} D_{1}+p_{2} D_{2}$. Hence, the VACFs or diffusion coefficients of the ions in RTILs could be expressed as a linear combination (with the corresponding weights) of the VACFs or diffusion coefficients of each state. Such an approximation is verified in Fig. 5(b) for the VACFs of cations (similar results for anions are shown in the Supplemental Material [108], Fig. S5).

The above analysis for VACFs supports the conjecture that the molecular kinetics in RTILs may be understood in terms of a two-state model with interstate exchange. Moreover, one can also conclude that the "dynamic decoupling" for the in-state molecular motion is observed in the sense that the timecorrelation functions in the free and bound states are statistically independent. Indeed, as it follows from Eq. (10), the total time-correlation function may be expressed as a weighted sum of two independent functions - one for the free motion and another for the bound one.

\section{E. Conductivity of RTILs}

Similar to the diffusion coefficient in Eq. (5), the conductivity of RTILs can be calculated from the time integral of the electric current autocorrelation function (ECACF); see Appendix E. The MD-obtained ECACFs decay quickly, within a few picoseconds, to zero [Fig. 6(a)], and the conductivity spectrum shows a higher peak shifting toward low frequency as the temperature increases [Fig. 6(b)]. More details for all studied temperatures are presented in Fig. S6 of the Supplemental Material [108].

Integrating the ECACF over time (Fig. S7 in the Supplemental Material [108]), one obtains the molar electrical conductivity $\Lambda$, which may be compared with the experimental data for [Bmim][TFSI] in Ref. [86]. Since only free ions participate in dc electrical current (bound ions in neutral clusters cannot carry a charge), one obtains (see Appendix E for details)

$$
\Lambda=\frac{N_{A}}{k_{B} T}\left(p_{+} q_{+}^{2} D_{+}+p_{-} q_{-}^{2} D_{-}\right)+\Lambda_{\text {cross }},
$$

where $N_{A}$ is Avogadro's number, $p_{+}$and $p_{-}$are, respectively, the fractions of cations and anions in the free state, $q_{+}$and $q_{-}$are their charges, $D_{+}$and $D_{-}$are their diffusion coefficients, and $\Lambda_{\text {cross }}$ quantifies cross-correlations in the motion of different free ions. We argue that the cross-correlation term is small and hence may be neglected (Appendix E). As a result, we arrive at a modified NernstEinstein equation based exclusively on the free ions:

$$
\Lambda_{\mathrm{NE}, \text { modified }}=\frac{N_{A}}{k_{B} T}\left(p_{+} q_{+}^{2} D_{+}+p_{-} q_{-}^{2} D_{-}\right) .
$$

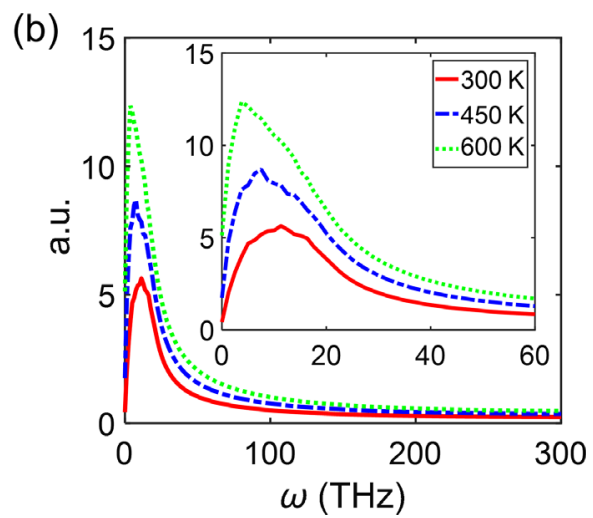

FIG. 6. The impact of the temperature on (a) the ECACF and (b) spectrum of frequency-dependent electrical conductivity. The data are obtained from MD simulations of RTIL [Bmim][TFSI]. 


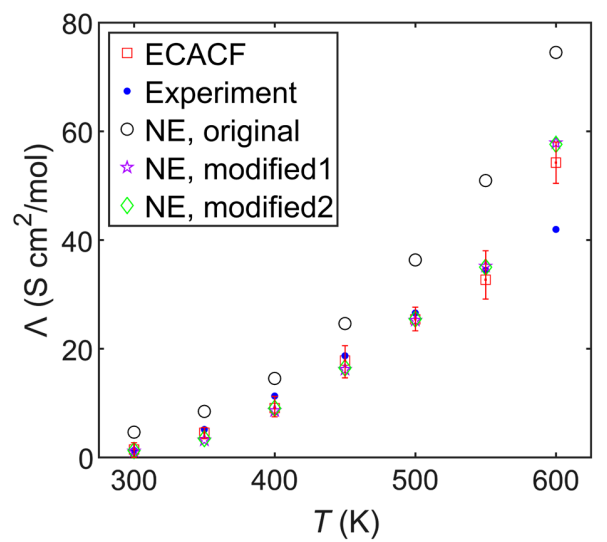

FIG. 7. The molar electrical conductivity $\Lambda$ of RTIL [Bmim] [TFSI] as a function of temperature. Red squares show the values calculated from the MD-simulated electric current autocorrelation function [Fig. 6(a)]. Blue dots are experimental data from Ref. [86]. Black circles display the values computed via original NernstEinstein equation (see Ref. [50]) with the overall diffusion coefficients of cations and anions [shown in Fig. 4(b)]. Purple stars are based on Eq. (12) (modified Nernst-Einstein equation), with the free-ion percentage evaluated by the trajectory-density method, while the green diamonds are the results of application of the ion-pairing method.

In Fig. 7, we compare the experimental conductivity for [Bmim][TFSI] from Ref. [86] with that computed from the time integral of the ECACF and from the modified NernstEinstein equation (12) based on free ions. Here we use the values $p_{i}$ obtained in the trajectory analysis, as well as the quantities found by the ion-pairing method. Figure 7 demonstrates excellent agreement (except for the last point of $600 \mathrm{~K}$, which is, actually, not a measured but theoretical value from a model with parameters fit to experimental data [86]) between the experimental data and results from the modified Nernst-Einstein equation based on the free ions as well as from ECACFs. Note that the conductivity obtained from the time integration of the ECACF contains the crosscorrelation term $\Lambda_{\text {cross }}$ [see Eqs. (11) and (E2) as well as the discussion in Appendix E]. Therefore, Fig. 7 indicates that good agreement between Eq. (12) and simulations can be achieved, suggesting that the cross term is indeed small and may be neglected with good accuracy. Finally, Fig. 7 also illustrates the consistency between evaluating the free-ion fraction through the trajectory-density and ion-pairing analyses.

The conclusion that the Nernst-Einstein equation should include only free ions could rationalize the findings of several research groups that the straightforward application of the Nernst-Einstein overestimates the RTIL conductivity $[50,58,87,88]$. Indeed, the use of the diffusion coefficients of the overall ions in Fig. 4(b), which account for all ion states, yields the conductivity that significantly exceeds the experimental value (black circles vs blue dots in Fig. 7) [89].

\section{F. Are the studied effects universal for other RTILs?}

To test the universality of the above phenomena, we perform MD simulations of another two RTILs with a different cation or anion (i.e., [Emim] $[\mathrm{TFSI}]$ and $[\mathrm{Bmim}]\left[\mathrm{PF}_{6}\right]$ ). Similar free-ion percentages by the trajectory-density and ion-pairing methods as well as the kinetic definition by Eq. (6) are observed for these two RTILs (Fig. S8 of the Supplemental Material [108]). Moreover, the same conclusion on conductivity drawn from [Bmim][TFSI] holds as well; that is, MDcomputed conductivities by Eq. (12) for [Emim][TFSI] and $[\mathrm{Bmim}]\left[\mathrm{PF}_{6}\right]$ are well in accord with the experimental data in Refs. [76,91], respectively (Fig. S9 of the Supplemental Material [108]).

\section{CONCLUDING REMARKS}

\section{A. Mechanisms of ion transport in RTILs}

There were different ideas about the mechanisms of ion transport in RTILs. One of the scenarios was proposed by Abbot $[92,93]$ who assumed that in such a concentrated ionic systems, only a hole mechanism of transport would be feasible. The findings presented above cannot directly prove or disprove that hypothesis, as looking at any individual trajectory, we cannot tell how each elementary step of ion transfer proceeds. Another related and often discussed scenario is a "quasi-Grotthuss" relay mechanism in which in each elementary act, an ion does not move far but shifts slightly, kicking another one to continue the motion and so on. Whereas this mechanism is not excluded for ions inside the clusters, our free ions seem to be able to move over considerable distances, as we can see it from their individual trajectories.

\section{B. "Ionic semiconductor" concept}

As is typical in condensed matter physics, in complex cases, people indulge in the language of "collective" phenomena. It is thus tempting to treat RTILs as an "ionic semiconductor" in which in the ground-state ions populate the ionic "valence" (or "excitonic") band, but they can be excited into a "conductance" band [94]. The band-gap energy that we obtain in the fits of Fig. 2(f) is, however, rather small $E_{g}=0.026 \mathrm{eV}$ (i.e., approximately $1 k_{B} T$ at room temperature).

An ionic semiconductor analogy for RTILs was raised in Ref. [7] and in the context of discussion of the results of Ref. [13]; our findings show that such a concept makes sense as an approximation to a complex problem. A similar idea was debated in the 1970s-1980s in an interpretation of ion transport in superionic conductors-single-chargecarrier solid electrolytes $[95,96]$. For instance, in $\mathrm{Ag}_{4} \mathrm{RbI}_{5}$ the conducting ions are silver cations, the rest of the ions forming a solid lattice in which $\mathrm{Ag}^{+}$ions move. But the latter are speculated to occupy two states: a ground immobile state in the crystal unit cells and an excited "conduction-band" state in which they move collectively. 
The band gap between them determines the activation energy of conductance that has a clear Arrhenius dependence in a wide range of temperatures with small activation energy of $0.069 \mathrm{eV}$ [97]. Of course, RTILs are much more complex systems than crystalline superionics: There is no lattice in RTILs, both ions are capable of moving, but the picture of long trajectories in the motion of free ions does not contradict the ionic semiconductor concept. Although we trace the path of an individual ion, its trajectory is a part of a cooperative motion of all ions, reflecting collective ion dynamics of the entire RTIL.

More experiments are needed to verify this concept. Measurements of the Hall effect come to mind first. However, the latter are difficult from an experimental point of view $[98,99]$, as well as they are not straightforward in interpretation $[100,101]$.

\section{Free and bound ions and the structure of EDL}

If the exchange between the conduction and valence bands is much faster than the $R C$ time of charging the double layers, which is practically always the case, it would be legitimate to speak about the average concentration of mobile charge carriers that contribute to the EDL formation. But if even only $15 \%$ of ions are, on average, free and give the major contribution to the EDL, it is already a concentrated ionic system. The spatial structure of the EDL if described in all its complexity will presumably contain an oscillating ("overscreening") part and the exponential tail ("underscreening") part; furthermore, bound ions will contribute not only to the effective dielectric constant of the system but also to the oscillating spatial potential and charge distributions. A recent study of the temperature dependence of the differential capacitance found the shape of the capacitance curve changes as if ion pairs and clusters were breaking up with increasing temperature [41]; this agrees with the findings of the present work.

\section{Underscreening paradox}

So far, the observations of massive underscreening in RTILs have been at least partially related with the ultralow concentration of free ions. Such low concentrations, however, are not approved by the findings of this paper, which reveal much larger concentrations compatible with the experimentally observed conductivities. Hence, the underscreening paradox remains unresolved and more work is needed to rationalize this effect. However, independent from the question of underscreening, our study provides insight into the nature of ion dynamics and conductivity in RTILs.

\section{ACKNOWLEDGMENTS}

We thank Fernando Bresme, Gleb Oshanin, Susan Perkin, Igor Sokolov, Yuri Budkov, and Tom Welton for useful discussions. G. F., M. C., and S. B. acknowledge the funding support from the National Natural Science Foundation of China (Grants No. 51876072 and No. 51836003) and the Shenzhen Basic Research Project (Grant No. JCYJ20170307171511292). A. A. K. acknowledges the Leverhulme Trust for funding (Grant No. RPG2016-223). M. U. acknowledges the financial support of the Deutsche Forschungsgemeinschaft Grant No. BA1008/21-1 and the Israel Science Foundation Grant No. 1141/18. N. B. acknowledges the financial support from the Russian Foundation for Basic Research, under Grant No. 18-2919198. Z.G. is supported through a studentship in the Centre for Doctoral Training on Theory and Simulation of Materials at Imperial College London funded by the EPSRC (Grant No. EP/L015579/1) and from the Thomas Young Centre under Grant No. TYC-101. Collaboration between Imperial College and Huazhong University of Science and Technology (HUST) is facilitated by the HUST Advisory Professorship to A. A. K. The computation of this work is completed using Tianhe II supercomputer in National Supercomputing Center in Guangzhou.

\section{APPENDIX A: MOLECULAR DYNAMICS SIMULATIONS}

As shown in Fig. 1, MD simulation of RTIL [Bmim] [TFSI] is performed using a customized MD code GROMACS [102]. The force fields of [Bmim][TFSI] are adopted from Ref. [103] by Borodin, neglecting their polarizability. It is worth mentioning that although these force fields are developed accounting for ion polarization, Borodin and co-workers [104] have also shown that the nonpolarizable version of these force fields could reproduce the bulk properties of RTILs as good as the polarizable one. A three-dimensional periodic cubic simulation system consisting of 300 ion pairs is simulated, with the temperature maintained in the interval of 300-600 K (in steps of $50 \mathrm{~K}$ ) using the Nose-Hoover thermostat with a relaxation time of $0.2 \mathrm{ps}$. The Parrinello-Rahman barostat is taken to maintain a pressure of $1 \mathrm{bar}$ with a relaxation time of $2 \mathrm{ps}$ in the isothermal-isobaric $(N-P-T)$ ensemble. The nonbonded interactions with repulsion and dispersion are computed using the Buckingham potential. The electrostatic interactions are computed using the particle mesh Ewald method. To compute the interactions in the reciprocal space, a fast-Fourier-transformation grid spacing of $0.12 \mathrm{~nm}$ and cubic interpolation for charge distribution are used. A cutoff distance of $1.2 \mathrm{~nm}$ is used in the calculation of electrostatic interactions in real space. The simulation box is initially equilibrated for $10 \mathrm{~ns}$ in the $N-P-T$ ensemble followed by a canonical ensemble $(N-V-T)$ for a 10-ns production run for trajectory analysis. To ensure the accuracy of the simulation results, each case is repeated three times with different initial configurations.

Additional MD simulations containing, respectively, 150 and 600 ion pairs of RTIL [Bmim][TFSI] together with those having 300 ion pairs throughout this study 
(a)

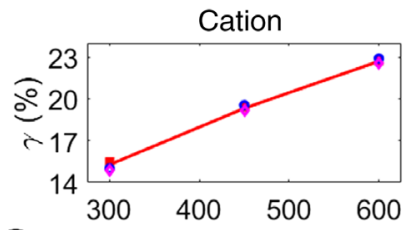

(b)

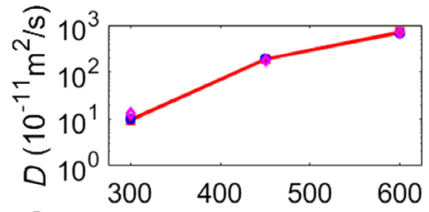

(c)

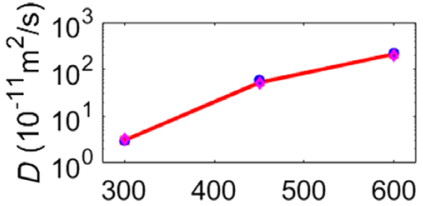

(d)

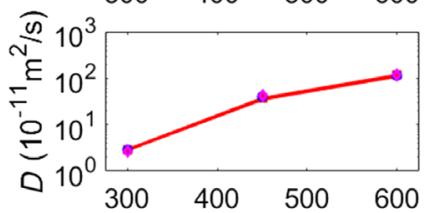

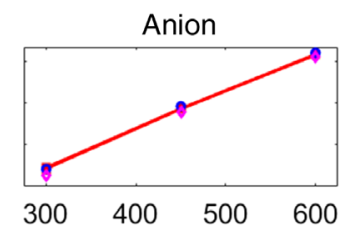
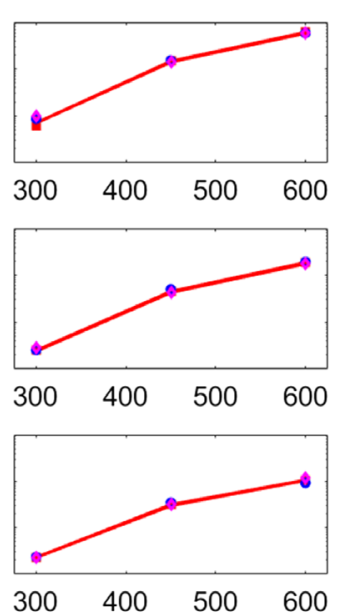

(e)

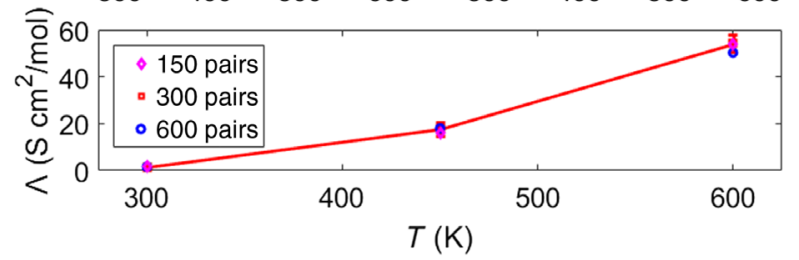

FIG. 8. The influence of MD simulation box size on (a) the free-ion percentage, (b) diffusion coefficient of ions in the free state, (c) diffusion coefficient of ions in the overall state, (d) diffusion coefficient of ions in the bound state, and (e) RTIL conductivity.

are performed to test whether the simulation box size affects the results. As shown in Fig. 8 and Fig. S10 in the Supplemental Material [108], it can be found that the simulation box size has ignorable influence on the results. Moreover, to evaluate the possible universality of the conclusions obtained from the modeling of RTIL [Bmim][TFSI], another two RTILs ([Emim][TFSI] and $[\mathrm{Bmim}]\left[\mathrm{PF}_{6}\right]$ ) are investigated with the same MDsimulation setup where [Emim][TFSI] has the same force fields as [Bmim][TFSI] from Ref. [103], but for $\left[\mathrm{Bmim}^{\mathrm{B} m}\left[\mathrm{PF}_{6}\right]\right.$ different force fields are adopted from Ref. [53].

\section{APPENDIX B: THE BOX-COUNTING METHOD}

The LTD is defined by the number of trajectory points of an ion (cation or anion) in the cubicle (a unit element of a space grid) divided by its volume. In this respect, two questions can arise: (i) What is the optimal size of the cubicle $l_{\text {cub }}$ ? (ii) Does the trajectory density depend on the trajectory length?

From the visual analysis of the ions' trajectories [Figs. 2(a) and 2(b) in the main text], we see that the tumbling motion alternates with the motion resembling longer flights. With the box-counting method, we wish to discriminate these two kinds of motions, that is, to locate the areas of tumbling and the areas of flights. To locate the tumbling areas, one should take $l_{\text {cub }}$ being roughly the same size as the tumbling area - this will yield a high density of trajectory points there. To distinguish the tumbling areas from the flight areas, $l_{\text {cub }}$ should be smaller than the characteristic length of the flight $L_{\text {flight }}$. Figure 9 shows the size distribution of different clusters of the ion trajectory cloud [Figs. 2(a) and 2(b)] in the bound state. One can see that the size of the area associated with the tumbling motions (the "cloud size") for the most trajectories is centered at around $0.3 \mathrm{~nm}$. Therefore, we adopt $0.3 \mathrm{~nm}$ as the optimal cubicle size for the box-counting method.

Should, generally, (and if "yes"-when) the LTD depend on the trajectory length? To answer this question, we use the two-state model discussed in the main text, focusing on the LTD of tumbling motion that corresponds to the bound ions. Let $\Delta t$ be the time interval between successive points of a trajectory, and then $\tau_{2} / \Delta t$ is the number of trajectory points corresponding to the average residence time $\tau_{2}$ of an ion in the bound state (see the main text for the definitions). The average LTD of the tumbling motion is, if we do not take into account reentrant trajectories, estimated as $\rho_{\text {tum }}^{(0)}=\left(\tau_{2} / \Delta t\right) / \delta V_{\text {bound }}$, where $\delta V_{\text {bound }}=(4 \pi / 3)\left(D_{2} \tau_{2}\right)^{3 / 2}$ is the average volume of the trajectory cloud associated with a bound ion, $D_{2}$ is the diffusion coefficient, and the quantity of dimensionality of

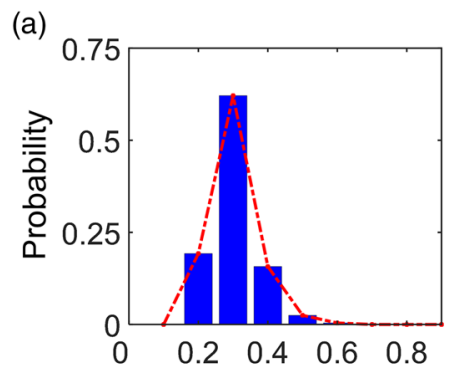

(b)

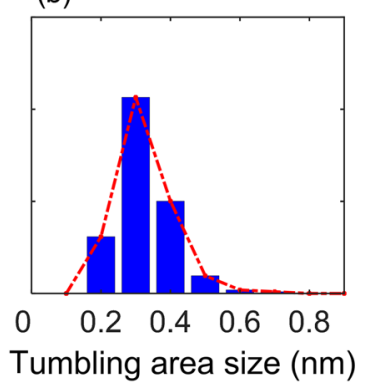

(c)

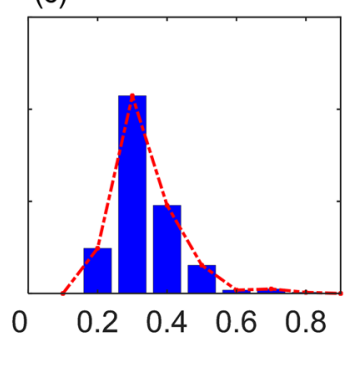

FIG. 9. For small enough cubicles, the distribution of sizes of the tumbling areas as obtained in the box-counting method weakly depends on the cubicle size $l_{\text {cub }}$ : (a) $0.2 \mathrm{~nm}$, (b) $0.3 \mathrm{~nm}$, and (c) $0.4 \mathrm{~nm}$. 
length $\sqrt{D_{2} \tau_{2}}$ estimates the average size of such a cloud. However, if the diffusive trajectory returns and gets back to a point inside the volume $\delta V_{\text {bound }}$, the LTD will be larger: It will double by one return, triple for two returns, etc. Let $P_{k}(t)$ denote the probability of exactly $k$ returns to this volume if the length of the trajectory is $t$ (for the simplicity of notations, we use the continuum time here). Then we obtain for the total LTD of the tumbling motion

$$
\begin{aligned}
\rho_{\mathrm{tum}} & =\rho_{\mathrm{tum}}^{(0)} P_{0}(t)+\rho_{\mathrm{tum}}^{(0)} P_{1}(t)+2 \rho_{\mathrm{tum}}^{(0)} P_{2}(t)+\ldots \\
& =\rho_{\mathrm{tum}}^{(0)}\left[P_{0}(t)+\sum_{k=1}^{k_{\max }} k P_{k}(t)\right],
\end{aligned}
$$

where $k_{\max }$ is the number of maximal possible returns during the time $t$. We analyze the case of long enough trajectories when $k_{\max } \gg 1$. In this case, it is reasonable to consider a simplified average model of an ion motion comprised of a series of alternating tumbling and free states, with the duration of all tumbling states $\tau_{2}$ and of all free states $\tau_{1}$.

The term "return" still needs some quantification. Suppose an ion starts a tumbling motion near the origin within the volume $\delta V_{\text {bound }}$. If after a sequence of meandering between the tumbling and free states, its trajectory ends somewhere inside the above volume $\delta V_{\text {bound, }}$, we call this event a return. If this return happens just after the first free state, which follows the initial tumbling state, we call this a "first return." According to our simplified model, the first return happens after the time equal to $\tau=\tau_{1}+\tau_{2}$. Then the minimal time for the second return will be $2 \tau$ and for the $k$ th return $k \tau$. Hence, the estimate of the maximal number of returns reads $k_{\max }=t / \tau$ (of course, the integer part of the quantity is implied).

Let us introduce $F(t)$ as the probability of the first return to the origin at time $t$ (see Ref. [105]). Accounting for that function, one can generally write nonlinear integral relationships between the $P_{0}(t)$ and $P_{k}(t)$ functions:

$$
P_{1}(t)=\int_{0}^{t} P_{0}\left(t-t_{1}\right) F\left(t_{1}\right) d t_{1}
$$

and

$$
P_{k}(t)=\int_{0}^{t} P_{0}\left(t-t_{1}\right) d t_{k} \ldots \int_{0}^{t_{2}} P_{0}\left(t_{2}-t_{1}\right) F\left(t_{1}\right) d t_{1} .
$$

First, we define the Laplace transform, with the Laplace transform parameter $s$ having dimensionality of inverse time as Eq. (B4)

$$
\tilde{\Phi}(s)=\int_{0}^{\infty} \Phi(t) e^{-s t} d t .
$$

Since $P_{1}(t)$ and $P_{k}(t)$ are convolution integrals, the Laplace transforms of these functions are related algebraically: $\tilde{P}_{1}(s)=\tilde{P}_{0}(s) \tilde{F}(s)$ and $\tilde{P}_{k}(s)=\tilde{P}_{0}(s)[\tilde{F}(s)]^{k}$. Hence, the Laplace transform of the LTD in Eq. (B1) reads

$$
\begin{aligned}
\tilde{\rho}_{\text {tum }}(s) & =\rho_{\text {tum }}^{(0)} \tilde{P}_{0}(s)\left[1+\sum_{k=1}^{k_{\max }} k[\tilde{F}(s)]^{k}\right] \\
& =\rho_{\text {tum }}^{(0)} \tilde{P}_{0}(s)\left[1+\frac{\tilde{F}(s)}{[1-\tilde{F}(s)]^{2}}\right],
\end{aligned}
$$

where we assume that $k_{\max }$ is large enough so that one can neglect the term with the factor $[\tilde{F}(s)]^{k_{\max }}$ [note that $\tilde{F}(s)<1$; see Ref. [105] ].

To understand the behavior of $\rho_{\text {tum }}(t)$ at $t \rightarrow \infty$, we need to explore the behavior of $\tilde{\rho}_{\text {tum }}(s)$ for $s \rightarrow 0$. Following Ref. [105] but restoring the dimensional format of the expression for $F(t)$, we get $\tilde{F}(s)=R-(1-R)^{2} \tau_{0}^{1 / 2} s^{1 / 2} /$ $(4 \pi)$. Here the constant $R$ is the probability of returning to the origin in infinite time, and the constant $\tau_{0}$ has the dimensionality of time. This characteristic time is related to the diffusion process; for the lattice model with discrete time, it corresponds to the time between the elementary acts of ion transfer [106].

In $1 \mathrm{D}$ and $2 \mathrm{D}$, the probability of a diffusion trajectory to return to the starting point in infinite time is 1 (i.e., $R=1$ ), which is also called as the "return theorem" [105]. In contrast, for 3D diffusion this probability is less than 1 (i.e., $R<1$ ) [105]. A specific value of $R$ depends on the lattice type for diffusion on a lattice or specific features of the off-lattice diffusion, but generally, $R$ is around 0.5 .

Using now $\tilde{P}_{0}(s)=(1-R) / s+(1-R)^{2} \tau_{0}^{1 / 2} /\left(4 \pi s^{1 / 2}\right)$ following from the relation $P_{0}(t)=1-\int_{0}^{t} F(t) d t$, we obtain from Eq. (B5) the small-s asymptotic expansion of $\tilde{\rho}_{\text {tum }}(s)$ :

$\tilde{\rho}_{\text {tum }}(s)=\rho_{\text {tum }}^{(0)}\left[\left(1+\frac{R^{2}}{1-R}\right) \frac{1}{s}+\frac{R(R-2)}{4 \pi}\left(\frac{\tau_{0}}{s}\right)^{1 / 2}+\ldots\right]$.

The inverse Laplace transform of Eq. (B6) yields the large time behavior of the LTD:

$\rho_{\text {tum }}(t)=\rho_{\text {tum }}^{(0)}\left[\left(1+\frac{R^{2}}{1-R}\right)-\frac{R(2-R)}{4 \pi^{3 / 2}}\left(\frac{\tau_{0}}{t}\right)^{1 / 2}\right]$.

For the case of no reentrant trajectories (i.e., when $R=0$ ), Eq. (B7) gives an obvious result: $\rho_{\text {tum }}(t)=\rho_{\text {tum }}^{(0)}$. In order to measure the actual value of $R$ for the studied system, one needs to collect statistics for the reentrant trajectories. 
But for a conservative estimate of the effect of the trajectory length on the LTD, we can just rewrite Eq. (B7) as

$$
\rho_{\text {tum }}=\rho_{\text {tum }}^{(0)}\left(1+\frac{R^{2}}{1-R}\right)\left[1-f(R)\left(\frac{\tau_{0}}{t}\right)^{1 / 2}\right],
$$

where the factor

$$
f(R)=\frac{R(2-R)}{4 \pi^{3 / 2}} /\left(1+\frac{R^{2}}{1-R}\right)
$$

quantifies the relative impact of the trajectory length $t$ on the LTD.

The maximal impact would be, of course, when $f(R)$ attains its maximum. The rhs of Eq. (B9) reaches maximum at $R=0.441$, with $f(0.441)=0.023$. For this case, Eq. (B8) reads

$$
\rho_{\mathrm{tum}}=\rho_{\mathrm{tum}}^{(0)} 1.348\left[1-\frac{0.023}{\sqrt{t / \tau_{0}}}\right] .
$$

Equation (B10) gives a conservative estimate of the largest possible effect of simulation time $t$ on the LTD.

Equations (B8) and (B10) quantify the "trajectory length" as $t / \tau_{0}$. But what could be the value of $\tau_{0}$ for our off-lattice model, if for the lattice model it equals the time between the successive elementary acts of ion transfer? We believe that the most conservative estimate would be $\tau_{0}=\tau=\tau_{1}+\tau_{2}$, which is the largest characteristic timescale related to the diffusive motion in our system.

Equation (B10) suggests that for sufficiently long trajectories, when $t / \tau \gg 1$, the impact of the trajectory length on the LTD is negligible. In our MD simulation, the trajectory length is $10 \mathrm{~ns}$, whereas $\tau$ is computed to be always smaller (or significantly smaller) than $100 \mathrm{ps}$ [see Fig. 3(b)], and we indeed see the independence of
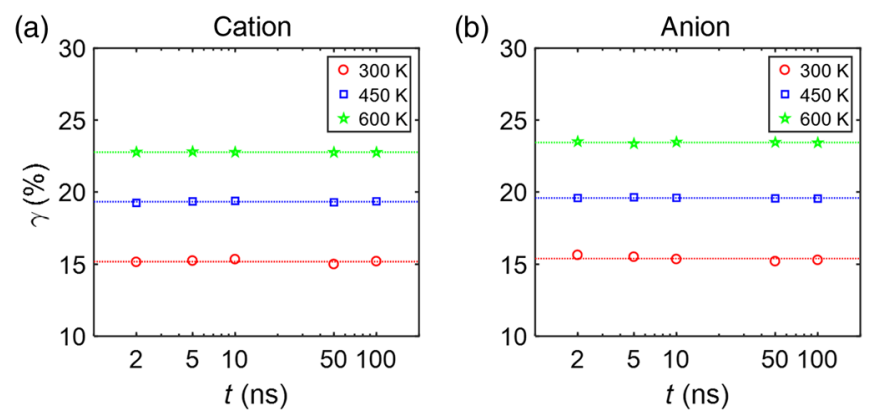

FIG. 10. Free-ion percentage $\gamma$ of (a) cations and (b) anions in [Bmim][TFSI] at 300, 450, and $600 \mathrm{~K}$ for different trajectory lengths. Dots for each temperature represent the average values for different running times of the MD simulation. The lines are a guide to the eye. One can clearly see a lack of dependence of $\gamma$ on the trajectory length for $t \geq 2$ ns (very tiny deviations from a constant $\gamma$ have a statistical nature). the LTD on the trajectory length. The direct evidence is as follows.

Since LTD has a statistical nature, the only meaningful way to check such a dependence is to analyze some average characteristics of the LTD. For instance, it may be the relative fraction of domains with a high trajectory density (e.g., larger than the average density) or the relative fraction of domains with a small trajectory density (e.g., smaller than the average density). The latter quantity is nothing but the percentage of free ions $\gamma$. Hence, we can now check the effect of the trajectory length on $\gamma$.

Simulations are done for three different temperatures for the system of 300 ion pairs with the trajectory length up to $100 \mathrm{~ns}$. The free-ion percentage $\gamma$ is computed at different trajectory lengths (Fig. 10), showing that $\gamma$ varies little with the trajectory length and thus demonstrating the independence of the LTD on the trajectory length.

\section{APPENDIX C: EQUILIBRIUM FRACTION OF FREE IONS}

We adopt the standard free-energy functional [84] for a field-free system, with the density of positive $n_{+}(\vec{r})$, negative $n_{-}(\vec{r})$ ions, and neutral clusters $n_{0}(\vec{r})$ supplemented by the conditions of local electroneutrality $n_{+}(\vec{r})=$ $n_{-}(\vec{r})$ and space-uniform packing fraction $\eta(\vec{r})=\eta_{0}$,

$$
n_{+} v_{+}+n_{-} v_{-}+n_{0} v_{0}=\eta_{0}
$$

where $n_{+}+n_{-}$is the number density of free ions (cations and anions), and $v_{+}, v_{-}$, and $v_{0}$ are, respectively, the molecular volume of a cation and anion, and a volume of an average neutral cluster. With these two constraints, the free energy of the system $F\left(n_{+}, n_{-}, n_{0}\right)$ reads

$$
\begin{aligned}
\beta F & =\int d \vec{r} n_{+}\left[\ln \left(\Gamma_{+}^{3} Z_{+} n_{+}\right)-1\right]+\int d \vec{r} n_{-}\left[\ln \left(\Gamma_{-}^{3} Z_{-} n_{-}\right)-1\right] \\
& +\int d \vec{r} n_{0}\left[\ln \left(\Gamma_{0}^{3} Z_{0} n_{0}\right)-1\right]+\beta \int d \vec{r} f_{e x}\left(n_{+}, n_{-}, n_{0}\right) \\
& +\lambda_{1} \int d \vec{r}\left(n_{+}-n_{-}\right)+\lambda_{2} \int d \vec{r}\left(n_{+} v_{+}+n_{-} v_{+}\right. \\
& \left.+n_{0} v_{0}-\eta_{0}\right),
\end{aligned}
$$

where $\beta=\left(k_{B} T\right)^{-1}, f_{\text {ex }}\left(n_{+}, n_{-}, n_{0}\right)$ is the nonideal (excess) part of the free energy, $\lambda_{1}$ and $\lambda_{2}$ are the Lagrange multipliers, $\Gamma_{+}, \Gamma_{-}$, and $\Gamma_{0}$ are, respectively, the thermal Broglie wavelengths of the positive ions, negative ions, and neutral aggregates treated as single composite particles, and $Z_{+}, Z_{-}$, and $Z_{0}$ are the according internal partition functions associated with these particles, which are comprised of many different atoms (see, e.g., Fig. 1).

The minimization of Eq. (C2) with respect to the concentrations $n_{+}(\vec{r}), n_{-}(\vec{r})$, and $n_{0}(\vec{r})$, which may be written as $\delta F\left(n_{+}, n_{-}, n_{0}\right) / \delta n_{+}=\delta F\left(n_{+}, n_{-}, n_{0}\right) / \delta n_{-}=$ $\delta F\left(n_{+}, n_{-}, n_{0}\right) / \delta n_{0}=0$, yields 


$$
\begin{array}{r}
\ln \left(\Gamma_{+}^{3} Z_{+} n_{+}\right)+\beta \mu_{+}+\lambda_{1}+\lambda_{2} v_{+}=0 \\
\ln \left(\Gamma_{-}^{3} Z_{-} n_{-}\right)+\beta \mu_{-}-\lambda_{1}+\lambda_{2} v_{-}=0, \\
\ln \left(\Gamma_{0}^{3} Z_{0} n_{0}\right)+\beta \mu_{0}+\lambda_{2} v_{0}=0,
\end{array}
$$

where $\mu_{\alpha}=\partial f_{\mathrm{ex}} / \partial n_{\alpha}$ is the excess part of the chemical potential and $\alpha=+, 0,-$. Solving the above Eq. (C3) together with the above two constraints, we obtain

$$
n_{+}=\frac{1}{\zeta} n_{0}^{\theta} e^{-\left(\mu_{f}-\theta \mu_{0}\right) / k_{B} T},
$$

where $\zeta=\left[\left(\Gamma_{+}^{3} Z_{+} \Gamma_{-}^{3} Z_{-}\right)^{1 / 2} /\left(\Gamma_{0}^{3} Z_{0}\right)^{\theta}\right], \theta=\left(v_{+}+v_{-}\right) / 2 v_{0}$, and $\mu_{f}=\left(\mu_{+}+\mu_{-}\right) / 2$. From Eq. (C4) follows the fraction of the free ions

$\gamma=\frac{n_{+}+n_{-}}{n_{+}+n_{-}+n_{0}}=\left[1+\frac{1}{2} \zeta n_{0}^{1-\theta} e^{\left(\mu_{f}-\theta \mu_{0}\right) / k_{B} T}\right]^{-1}$.

Now we apply the general thermodynamic relation for the chemical potential (actually, to its excess part),

$$
\mu=u-T s+v \delta p,
$$

where $u$ and $s$ are, respectively, the excess part of the internal energy and entropy per particle, $v$ is the system volume per particle, and $\delta p$ is the nonideal part of pressure. If for a moment we use an extreme simplification of a "lattice model," in which a site can be occupied by a cation, anion, or a cluster, then $v_{+}=v_{-}=v_{0}$. In this case, $\theta=1$ and we obtain a simpler relation, as obtained in Ref. [41]

$$
\gamma=\frac{1}{1+\frac{1}{2} \zeta e^{\left(E_{g}-T \Delta S_{\mathrm{ex}}\right) / k_{B} T},}
$$

with $E_{g}=\frac{1}{2}\left[\left(u_{+}-u_{0}\right)+\left(u_{-}-u_{0}\right)\right]$ being the energy gap between the free and bound states, that is, the change of the internal energy per emerging free ion. $\Delta S_{\mathrm{ex}}=\frac{1}{2}\left[\left(s_{+}-s_{0}\right)+\right.$ $\left.\left(s_{-}-s_{0}\right)\right]$ is the according change of entropy.

To compute the partition functions $Z_{\alpha}$ with $\alpha= \pm, 0$, that are needed to obtain $\zeta$, one has to exploit some models, which is an interesting problem by itself but is beyond the scope of the present study. In the lattice model of Ref. [41], $\zeta$ was taken to be 1 (i.e., $\zeta=1$ ).

Equation (C7) may be written in the form $\ln [(1 / \gamma)-1]=\left\{\ln \zeta-\left[\ln 2+\left(\Delta S_{\text {ex }} / k_{B}\right)\right]\right\}+\left(E_{g} / k_{B} T\right)$ used in the main text, which allows us to find $E_{g}$ from the slope of the dependence of $\ln [(1 / \gamma)-1]$ on $(1 / T)$. Using $\zeta=1$, as in the lattice model of Ref. [41], one can find $\Delta S_{\text {ex }}$ from the intercept of the above dependence. $\Delta S_{\text {ex }}$ occurs to be slightly negative for this model for both cations and anions. This result seemingly indicates the deficiency of the lattice model to quantify the entropy change between the free and bound states, which is not surprising and related to the difficulty of lattice theories to describe the entropy of liquids [85].
For the general case of arbitrary volumes of the ions and clusters, Eq. (C5) may be recast into the form

$$
\begin{aligned}
\ln \left(\frac{1}{\gamma}-1\right)= & {\left[\ln \zeta+(1-\theta) \ln n_{0}-\left(\ln 2+\frac{\Delta S_{\mathrm{ex}}}{k_{B}}\right)\right] } \\
& +\frac{E_{g}}{k_{B} T}
\end{aligned}
$$

where $\Delta S_{\text {ex }}=\frac{1}{2}\left[\left(s_{+}-\theta s_{0}\right)+\left(s_{-}-\theta s_{0}\right)\right]$ and $E_{g}=$ $\frac{1}{2}\left[\left(u_{+}-\theta u_{0}\right)+\left(u_{-}-\theta u_{0}\right)\right]$. Because of the logarithmically weak dependence of the quantity in the square brackets of the right-hand side of Eq. (C8) on temperature, one can treat this as a constant and find the value of $E_{g}$ from the slope of the dependence of $\ln [(1 / \gamma)-1]$ vs $(1 / T)$. Moreover, without loss of generality, one can choose $u_{0}=0$ that is to consider the excess internal energy of neutral clusters as zero. Then, $E_{g}=\frac{1}{2}\left(u_{+}+u_{-}\right)-u_{0}$ is again the energy gap between the free and bound states of the ions. Therefore, the lattice model from Ref. [41] with $\theta=1$ yields the same result for the energy gap between the free and bound states as a more general model.

\section{APPENDIX D: TIME-CORRELATION FUNCTIONS FOR TWO-STATE SYSTEM WITH EXCHANGE}

The general theory of time-correlation functions for multistate systems with exchange has been developed in Ref. [75]. Here we briefly outline the derivation for a case of an exchange process, which is not necessarily Poissonian, with the according adaptation of the theory to the two-state RTILs.

Consider a system where ions can successively belong to two states with different kinetic behaviors. In equilibrium systems, a dynamical balance between these two states is held. The exchange kinetic is quantified by the survival probability function $c(t)=[\langle h(0) h(t)\rangle /\langle h(0) h(0)\rangle]$, where $h(t)$ is the indicator of the presence of a particle in the same state without interstate transition (see the main text). $c(t)$ quantifies the probability of remaining at time $t$ in the same state (without leaving it) as at initial time $t=0$. We also introduce the related function $g(t)=-d c(t) / d t$; the quantity $g(t) d t$ gives the probability of a particle to remain up to time $t$ in the same state as in time $t=0$ and then leave this state during a (small) time interval $(t, t+d t)$. For a Poissonian process, $c(t)=e^{-t / \tau}$, where $\tau$ is the mean residence time in the state; in this case, $d c / d t=-c(t) / \tau$. If $c(t)$ is not exponential, the process is not Poissonian, and the memory effect may be described by the memory function. Specifically, an approach to describe the memory effect in two-state kinetics is related to the Fokker-Planck equation with a fractional time derivative [73]. It predicts a stretched exponent for the function $c(t)$, i.e., $c(t)=e^{-t^{\alpha}}$, where $\alpha$ is the exponent of the fractional derivative [73]. We wish to stress, however, 
that our results for $c(t)$ may be much better fitted by the double exponent rather than by a stretched exponent; moreover, the numerical solution of the Fokker-Planck equation with a fractional derivative does not demonstrate a stretched exponent [107].

In what follows, we derive time-correlation functions for a system with the interstate exchange for non-Poissonian processes. Consider a dynamic variable $A(t)$ which is a unitary quantity, i.e., $A A^{+}=1$, where $A^{+}$is the Hermitian conjugate of $A$. An example of such a quantity may be $A(t)=e^{i \vec{k} \cdot \vec{r}(t)}$, where $\vec{k}$ is the wave vector and $\vec{r}(t)$ is a particle radius vector. Let $\Phi(t)=\left\langle A^{+}(0) A(t)\right\rangle$ be the total time-correlation function for that quantity, while $\Phi_{i j}(t)$ is the partial correlation function associated with the ions located at state $i$ at time $t=0$ and occupying state $j$ at later time $t$. Then one can write

$$
\Phi(t)=p_{1}\left[\Phi_{11}(t)+\Phi_{12}(t)\right]+p_{2}\left[\Phi_{21}(t)+\Phi_{22}(t)\right] .
$$

To compute $\Phi_{i j}(t)$, we use the survival probability function and its derivative and additionally assume that the dynamics in the successive states is independent. At the same time, the dynamics in either state may be non-Markovian, as well as the transition process itself may also possess memory.

Introduce now function $\Phi_{11}^{(n)}(t)$, which is similar to $\Phi_{11}(t)$ but with the additional condition that an ion was in state 1 at $t=0$ and having underwent after that $n=2 k$ successive interstate transitions, returns at time $t_{n}<t$ back to the same state

$$
\begin{aligned}
\Phi_{11}^{(n)}(t)= & \int_{0}^{t} c_{1}\left(t-t_{n}\right) d t_{n} \int_{0}^{t_{n}} g_{2}\left(t_{n}-t_{n-1}\right) d t_{n-1}, \ldots, \\
& \times \int_{0}^{t_{2}} g_{1}\left(t_{1}\right) d t_{1}\left\langle A^{+}(0) A\left(t_{1}\right) A^{+}\left(t_{1}\right) A\left(t_{2}\right) A^{+}\left(t_{2}\right), \ldots,\right. \\
& \left.\times A^{+}\left(t_{n}\right) A(t)\right\rangle,
\end{aligned}
$$

where in the second line of Eq. (D2) we write $\left\langle A^{+}(0) A(t)\right\rangle$ using the property $A^{+} A=1$. We also average over all possible transition instants at $t_{1}, t_{2}, \ldots, t_{n}$ with the probability of changing the state $g_{1}\left(t_{1}\right), \ldots, g_{2}\left(t_{n}-t_{n-1}\right)$ and remain in the final state $c_{1}\left(t-t_{n}\right)$. Since the dynamics in the successive states is independent, we can perform an independent average for all states and then write

$$
\begin{aligned}
& \left\langle A^{+}(0) A\left(t_{1}\right) A^{+}\left(t_{1}\right) A\left(t_{2}\right) A^{+}\left(t_{2}\right), \ldots, A^{+}\left(t_{n}\right) A(t)\right\rangle \\
& \quad=\left\langle A^{+}(0) A\left(t_{1}\right)\right\rangle\left\langle A^{+}\left(t_{1}\right) A\left(t_{2}\right)\right\rangle, \ldots,\left\langle A^{+}\left(t_{n}\right) A(t)\right\rangle \\
& \quad=\phi_{1}\left(t_{1}\right) \phi_{2}\left(t_{2}-t_{1}\right), \ldots, \phi_{1}\left(t-t_{n}\right),
\end{aligned}
$$

with $\phi_{1}(t)$ and $\phi_{2}(t)$ being the partial time-correlation functions for the first and second states, that is, $\phi_{i}(t)=$ $\left\langle A^{+}(0) A(t)\right\rangle$ describes the time correlation function for a molecule that resides in the state $i(i=1,2)$ during the time interval $[0, t]$. One can notice that the right-hand side of Eq. (D2) is a convolution of the function $\Phi_{1}^{\prime \prime}(t)=\phi_{1}(t) c_{1}(t)$ and functions $\Phi_{1}^{\prime}(t)=\phi_{1}(t) g_{1}(t)$ and $\Phi_{2}^{\prime}(t)=\phi_{2}(t) g_{2}(t)$.

Now we find the Laplace transform [as defined in Eq. (B4)] of the correlation function. With the properties of the Laplace transform, Eqs. (D2) and (D3) yield

$$
\tilde{\Phi}_{11}^{(n)}(s)=\tilde{\Phi}_{1}^{\prime \prime}(s)\left[\tilde{\Phi}_{1}^{\prime}(s) \tilde{\Phi}_{2}^{\prime \prime}(s)\right]^{n / 2} .
$$

Summing over all $n=2 k$, from $k=0$ to $k=\infty$, we obtain $\tilde{\Phi}_{11}(s)=\tilde{\Phi}_{1}^{\prime \prime}(s)\left[1-\tilde{\Phi}_{1}^{\prime}(s) \tilde{\Phi}_{2}^{\prime}(s)\right]^{-1}$ and, similarly, $\tilde{\Phi}_{12}(s)=\tilde{\Phi}_{2}^{\prime \prime}(s) \tilde{\Phi}_{1}^{\prime}(s)\left[1-\tilde{\Phi}_{1}^{\prime}(s) \tilde{\Phi}_{2}^{\prime}(s)\right]^{-1}$. The functions $\tilde{\Phi}_{22}(s)$ and $\tilde{\Phi}_{21}(s)$ may be obtained from the above ones using the interchange of the indexes, $1 \leftrightarrow 2$. Hence, Eq. (D1) finally yields

$$
\tilde{\Phi}(s)=\frac{\left(p_{1} \tilde{\Phi}_{1}^{\prime \prime}+p_{2} \tilde{\Phi}_{2}^{\prime \prime}\right)+\left(p_{1} \tilde{\Phi}_{2}^{\prime \prime} \tilde{\Phi}_{1}^{\prime}+p_{2} \tilde{\Phi}_{1}^{\prime \prime} \tilde{\Phi}_{2}^{\prime}\right)}{1-\tilde{\Phi}_{1}^{\prime} \tilde{\Phi}_{2}^{\prime}}
$$

When the exchange process is Poissonian with $c_{1}(t)=$ $e^{-t / \tau_{1}}$ and $c_{2}(t)=e^{-t / \tau_{2}}$, Eq. (D5) can be reduced to the previously known result [75],

$$
\tilde{\Phi}(s)=\frac{p_{1} \tilde{\phi}_{1}\left(s+\tau_{1}^{-1}\right)+p_{2} \tilde{\phi}_{2}\left(s+\tau_{2}^{-1}\right)+2 p_{1} p_{2} \tau_{\mathrm{ex}}^{-1} \tilde{\phi}_{1}\left(s+\tau_{1}^{-1}\right) \tilde{\phi}_{2}\left(s+\tau_{2}^{-1}\right)}{1-p_{1} p_{2} \tau_{\mathrm{ex}}^{-2} \tilde{\phi}_{1}\left(s+\tau_{1}^{-1}\right) \tilde{\phi}_{2}\left(s+\tau_{2}^{-1}\right)}
$$

where $\tau_{\mathrm{ex}}^{-1}=\tau_{1}^{-1}+\tau_{2}^{-1}$.

A correlation time of a dynamical variable is defined through the time integral of its correlation function:

$$
\tau_{A}^{(1)}=\int_{0}^{\infty} \phi_{1}(t) d t=\tilde{\phi}_{1}(s=0)
$$

This quantity characterizes the time when correlations between the initial value of the variable $A$ and its value at time $t$ become negligible, that is, $\phi_{1}(t) \approx 0$ if $t \gg \tau_{A}^{(1)}$. For the Laplace transform, one can use the estimate $\tilde{\phi}_{1}(s) \sim \tau_{A}^{(1)}$. The mean residence time $\tau_{i}=\int_{0}^{\infty} c_{i}(t) d t$ that describes the characteristic time of the variation of the survival probability function may be also used to estimate $g_{i}$, that is, $g_{i}=-d c_{i} / d t \sim c_{i} / \tau_{i}$.

Consider the case of slow exchange, $\tau_{A}^{(i)} \ll \tau_{i}$. In this case, while the correlation function $\phi_{i}(t)$ decays to zero for $t>\tau_{A}^{(i)}$, the function $c_{i}(t)$ does not noticeably change, justifying the 
approximations $\quad \tilde{\Phi}_{i}^{\prime \prime}(s)=\int_{0}^{\infty} \phi_{i}(t) c_{i}(t) e^{-s t} d t \approx \int_{0}^{\infty} \phi_{i}(t) \times$ $e^{-s t} d t=\tilde{\phi}_{i}(s) \quad$ and $\quad \tilde{\Phi}_{i}^{\prime}(s)=\int_{0}^{\infty} \phi_{i}(t) g_{i}(t) e^{-s t} d t \sim$ $\int_{0}^{\infty} \phi_{i}(t)\left(c_{i} / \tau_{i}\right) e^{-s t} d t \sim \tilde{\phi}_{i}(s) / \tau_{i} \sim \tau_{A}^{(i)} / \tau_{i} \ll 1$.

Substituting these approximations into Eq. (D5), we obtain for the case of slow exchange,

$$
\begin{aligned}
\tilde{\Phi}(s) & =p_{1} \tilde{\phi}_{1}(s)+p_{2} \tilde{\phi}_{2}(s), \\
\Phi(t) & =p_{1} \phi_{1}(t)+p_{2} \phi_{2}(t) .
\end{aligned}
$$

To utilize the above theory for the VACF, we use the relations between the VACF $K_{v}(t)$ as introduced in the main text, with the self-part of the intermediate scattering function [74,84]. It is defined as $F_{s}(\vec{k}, t)=$ $\left\langle A^{+}(0) A(t)\right\rangle$, with $A(t)=e^{i \vec{k} \cdot \vec{r}(t)}$, where $\vec{r}(t)$ is a particle radius vector and $\vec{k}$ is the wave vector [74,84]. Namely,

$$
F_{s}(\vec{k}, t)=1-\frac{1}{3} \vec{k}^{2} \int_{0}^{t}(t-\tau) K_{v}(\tau) d \tau+O\left(k^{4}\right),
$$

and the inverse relation for the according Laplace transforms:

$$
\tilde{K}_{v}(s)=\lim _{k \rightarrow 0} 3\left[\frac{s}{k^{2}}-\frac{s^{2}}{k^{2}} \tilde{F}_{s}(\vec{k}, s)\right] .
$$

Using the Laplace transform of the first relation in Eq. (D8), one can express $\tilde{F}_{s}(\vec{k}, s)$ in terms of the Laplace transform of the velocity-correlation function and then find the according Laplace transforms of the functions $\tilde{\Phi}_{i}^{\prime}(t)$ and $\tilde{\Phi}_{i}^{\prime \prime}(t)$. Using these functions in Eq. (D5), one obtains the Laplace transform of $\tilde{F}_{s}(\vec{k}, s)=$ $\tilde{\Phi}(s)$ for the system with exchange. Substituting the results into Eq. (D9), one arrives after some algebra at Eq. (7). For the case of slow exchange $\tau_{A}^{(i)} \ll \tau_{i}$, one obtains Eq. (10) from Eqs. (D7) and (D9).

\section{APPENDIX E: CONDUCTIVITY COMPUTATION}

The linear response theory relates the specific directcurrent conductivity $(\sigma)$ and the autocorrelation function of an electric current $\vec{J}(t)=\sum_{m} q_{m} \vec{v}_{m}(t)$, where $q_{m}$ and $\vec{v}_{m}(t)$ are the charge and velocity of the $m$ th particle, respectively, through the Green-Kubo relation [84,88]

$$
\sigma=\frac{1}{3 V k_{B} T} \int_{0}^{\infty}\langle\vec{J}(0) \cdot \vec{J}(t)\rangle d t,
$$

where $V$ is the system volume, and the averaging is performed for the equilibrium system without external electric field; $\sigma$ is related to the molar conductivity $\Lambda$ as $\sigma=n_{\text {tot }} \Lambda / N_{A}$, where $n_{\text {tot }}$ is the total number density of ions and $N_{A}$ is Avogadro's number. With the above expression for the electric current, one can recast Eq. (E1) into the form

$$
\begin{aligned}
\sigma= & \frac{1}{3 V k_{B} T}\left[\sum_{m \in \text { free }} q_{m}^{2} \int_{0}^{\infty}\left\langle\vec{v}_{m}(0) \cdot \vec{v}_{m}(t)\right\rangle d t\right. \\
& \left.+\sum_{m \neq m^{\prime}} \sum_{m, m^{\prime} \in \text { free }} q_{m} q_{m^{\prime}} \int_{0}^{\infty}\left\langle\vec{v}_{m}(0) \cdot \vec{v}_{m^{\prime}}(t)\right\rangle d t\right],
\end{aligned}
$$

where the summation is to be performed over the free-ions only. The first term in the brackets in Eq. (E2) corresponds to the diffusion of free ions (cations and anions); see Eqs. (4) and (5). Hence,

$$
\sigma=\frac{N_{+}}{V} \frac{q_{+}^{2}}{k_{B} T} D_{+}+\frac{N_{-}}{V} \frac{q_{-}^{2}}{k_{B} T} D_{-}+\sigma_{\mathrm{cross}}
$$

where $N_{+}$and $N_{-}$are respectively the numbers of free cations and free anions in the system, and $\sigma_{\text {cross }}$ refers to the cross-correlation velocity terms. Taking into account that $n_{ \pm}=N_{ \pm} / V=p_{ \pm} n_{\text {tot }}$ as defined in the main text, $p_{+}$and $p_{-}$are, respectively, the fractions of cations and anions in the free state, we arrive at

$$
\Lambda=\Lambda_{+}+\Lambda_{-}+\Lambda_{\text {cross }}=\Lambda_{\mathrm{NE}, \text { modified }}+\Lambda_{\text {cross }}
$$

with $\Lambda_{ \pm}=N_{A} p_{ \pm} q_{ \pm}^{2} D_{ \pm} / k_{B} T$, and $\Lambda_{\text {cross }}$ is the crosscorrelation term that appears to be small when we compare the result with simulations.

The smallness of the cross term may be explained as follows: The dynamics of the $i$ th ion in dense RTILs is determined by interactions with many other particles, where most of them are neutral ion pairs or clusters. Hence, the influence of the $i$ th free ion on the $j$ th one is relatively small, which results in a small correlation function $\left\langle\vec{v}_{i}(0) \cdot \vec{v}_{j}(t)\right\rangle$. Moreover, two other effects make this term even smaller: the depletion of ion concentration for the case of ions of the same charge, and the fast recombination of closely located ions of the opposite sign that reduces the number of free ions.

[1] T. Welton, Room-Temperature Ionic Liquids. Solvents for Synthesis and Catalysis, Chem. Rev. 99, 2071 (1999).

[2] J.P. Hallett and T. Welton, Room-Temperature Ionic Liquids: Solvents for Synthesis and Catalysis. 2, Chem. Rev. 111, 3508 (2011).

[3] N. V. Plechkova and K. R. Seddon, Applications of Ionic Liquids in the Chemical Industry, Chem. Soc. Rev. 37, 123 (2008).

[4] M. C. Buzzeo, R. G. Evans, and R. G. Compton, NonHaloaluminate Room-Temperature Ionic Liquids in Electrochemistry-A Review, Chem. Phys. Chem. 5, 1106 (2004). 
[5] M. Galiński, A. Lewandowski, and I. Stępniak, Ionic Liquids as Electrolytes, Electrochim. Acta 51, 5567 (2006).

[6] D. S. Silvester and R. G. Compton, Electrochemistry in Room Temperature Ionic Liquids: A Review and Some Possible Applications, Z. Phys. Chem. 220, 1247 (2006).

[7] M. V. Fedorov and A. A. Kornyshev, Ionic Liquids at Electrified Interfaces, Chem. Rev. 114, 2978 (2014).

[8] A. A. Kornyshev, Double-Layer in Ionic Liquids: Paradigm Change? J. Phys. Chem. B 111, 5545 (2007).

[9] M. Z. Bazant, B. D. Storey, and A. A. Kornyshev, Double Layer in Ionic Liquids: Overscreening versus Crowding, Phys. Rev. Lett. 106, 046102 (2011).

[10] M. V. Fedorov, N. Georgi, and A. A. Kornyshev, Double Layer in Ionic Liquids: The Nature of the Camel Shape of Capacitance, Electrochem. Comm. 12, 296 (2010).

[11] Z. $\mathrm{Gu}$ and J.F. Brennecke, Volume Expansivities and Isothermal Compressibilities of Imidazolium and Pyridinium-Based Ionic Liquids, J. Chem. Eng. Data 47, 339 (2002).

[12] Y. Yu et al., Free Volume and Phase Transitions of 1-butyl-3-methylimidazolium Based Ionic Liquids from Positron Lifetime Spectroscopy, Phys. Chem. Chem. Phys. 14, 6856 (2012).

[13] M. A. Gebbie, H. A. Dobbs, M. Valtiner, and J. N. Israelachvili, Long-Range Electrostatic Screening in Ionic Liquids, Proc. Natl. Acad. Sci. U.S.A. 112, 7432 (2015).

[14] M. A. Gebbie, M. Valtiner, X. Banquy, E. T. Fox, W. A. Henderson, and J. N. Israelachvili, Ionic Liquids Behave as Dilute Electrolyte Solutions, Proc. Natl. Acad. Sci. U.S.A. 110, 9674 (2013).

[15] S. Perkin, M. Salanne, P. Madden, and R. Lynden-Bell, Is a Stern and Diffuse Layer Model Appropriate to Ionic Liquids at Surfaces? Proc. Natl. Acad. Sci. U.S.A. 110, E4121 (2013).

[16] A. M. Smith, A. A. Lee, and S. Perkin, The Electrostatic Screening Length in Concentrated Electrolytes Increases with Concentration, J. Phys. Chem. Lett. 7, 2157 (2016).

[17] M. A. Gebbie et al., Long Range Electrostatic Forces in Ionic Liquids, Chem. Commun. 53, 1214 (2017).

[18] A. A. Lee, C. S. Perez-Martinez, A. M. Smith, and S. Perkin, Underscreening in Concentrated Electrolytes, Faraday Discuss. 199, 239 (2017).

[19] H. K. Stassen, R. Ludwig, A. Wulf, and J. Dupont, Imidazolium Salt Ion Pairs in Solution, Chemistry 21, 8324 (2015).

[20] R. M. Adar, T. Markovich, and D. Andelman, Bjerrum Pairs in Ionic Solutions: A Poisson-Boltzmann Approach, J. Chem. Phys. 146, 194904 (2017).

[21] N. Gavish and A. Yochelis, Theory of Phase Separation and Polarization for Pure Ionic Liquids, J. Phys. Chem. Lett. 7, 1121 (2016).

[22] A. A. Lee, D. Vella, S. Perkin, and A. Goriely, Are RoomTemperature Ionic Liquids Dilute Electrolytes? J. Phys. Chem. Lett. 6, 159 (2015).

[23] Z. A. H. Goodwin and A. A. Kornyshev, Underscreening, Overscreening and Double-Layer Capacitance, Electrochem. Comm. 82, 129 (2017).

[24] Z. Hu and C.J. Margulis, Heterogeneity in a RoomTemperature Ionic Liquid: Persistent Local Environments and the Red-Edge Effect, Proc. Natl. Acad. Sci. U.S.A. 103, 831 (2006).

[25] There were a number of neutron and x-ray scattering studies [26-30] focused on the investigation of mesoscopic heterogeneity of ionic liquids caused by the presence of fatty tails of ions that cause a propensity to form mesophases, similar to solutions of amphiphiles and microemulsions. This happens with imidazolium-based cations, with the number of their chain elements greater than six. Recently, a homologous series of phosphonium ionic liquids were studied via $\mathrm{x}$-ray scattering, dynamic mechanical spectroscopy, and broadband dielectric spectroscopy, systematically varying alkyl chain lengths of the cation [31].

[26] K. Fujii, R. Kanzaki, T. Takamuku, Y. Kameda, S. Kohara, M. Kanakubo, M. Shibayama, S.-i. Ishiguro, and Y. Umebayashi, Experimental Evidences for Molecular Origin of Low-Q peak in Neutron/X-Ray Scattering of 1-alkyl-3-methylimidazolium bis(trifluoromethanesulfonyl)amide Ionic Liquids, J. Chem. Phys. 135, 244502 (2011).

[27] C. S. Santos, N.S. Murthy, G. A. Baker, and E. W. Castner, Jr., Communication: X-Ray Scattering from Ionic Liquids with Pyrrolidinium Cations, J. Chem. Phys. 134, 121101 (2011).

[28] O. Russina, A. Triolo, L. Gontrani, and R. Caminiti, Mesoscopic Structural Heterogeneities in Room-Temperature Ionic Liquids, J. Phys. Chem. Lett. 3, 27 (2012).

[29] K. Fujii, S. Kohara, and Y. Umebayashi, Relationship between Low-Q Peak and Long-Range Ordering of Ionic Liquids Revealed by High-Energy X-Ray Total Scattering, Phys. Chem. Chem. Phys. 17, 17838 (2015).

[30] H. Weiss, J. Mars, H. Li, G. Kircher, O. Ivanova, A. Feoktystov, O. Soltwedel, M. Bier, and M. Mezger, Mesoscopic Correlation Functions in Heterogeneous Ionic Liquids, J. Phys. Chem. B 121, 620 (2017).

[31] T. Cosby, Z. Vicars, M. Heres, K. Tsunashima, and J. Sangoro, Dynamic and Structural Evidence of Mesoscopic Aggregation in Phosphonium Ionic Liquids, J. Chem. Phys. 148, 193815 (2018).

[32] W. Kob, C. Donati, S. J. Plimpton, P. H. Poole, and S. C. Glotzer, Dynamical Heterogeneities in a Supercooled Lennard-Jones Liquid, Phys. Rev. Lett. 79, 2827 (1997).

[33] D. F. Kennedy and C. J. Drummond, Large Aggregated Ions Found in Some Protic Ionic Liquids, J. Phys. Chem. B 113, 5690 (2009).

[34] H. Weingärtner, Understanding Ionic Liquids at the Molecular Level: Facts, Problems, and Controversies, Angew. Chem., Int. Ed. Engl. 47, 654 (2008).

[35] H. Weingärtner, NMR Studies of Ionic Liquids: Structure and Dynamics, Curr. Opin. Colloid Interface Sci. 18, 183 (2013).

[36] Following $\mathrm{Hu}$ and Margulis [24], there were MD simulations exploring structural and dynamic heterogeneity of RTILs [37-40] associated with the investigation of hydrophobic and ionic domains in RTILs with cation alkyl chains of different lengths. The simulation in Ref. [37] has revealed relatively long-lived conformations that can appear when the aggregation of alkyl chains takes place within nonpolar domains, implying an intricate relationship between structural and dynamical heterogeneity in 
[Bmim]Cl. A recent systematic study [36] with gradual increase of the size of the hydrophobic part of the ammonium-based cations has demonstrated the expected enhancement of structural and dynamic heterogeneity with longer cationic chains and the overall asymmetry of the shape of cations. In Ref. [39], spatial clustering of "mobile" and "immobile" ions has been studied via fourpoint time-correlation functions that allowed access to both the length scale of correlated regions and the time associated with the correlation. The "mechanical heterogeneity" has been explored via simulations of local highfrequency elastic and shear moduli [40].

[37] S. M. Urahata and M. C. C. Ribeiro, Unraveling Dynamical Heterogeneity in the Ionic Liquid 1-butyl-3methylimidazolium Chloride, J. Phys. Chem. Lett. 1, 1738 (2010).

[38] K. Usui, J. Hunger, M. Bonn, and M. Sulpizi, Dynamical Heterogeneities of Rotational Motion in Room Temperature Ionic Liquids Evidenced by Molecular Dynamics Simulations, J. Chem. Phys. 148, 193811 (2018).

[39] J. Liu, J. A. L. Willcox, and H. J. Kim, Heterogeneous Dynamics of Ionic Liquids: A Four-Point Time Correlation Function Approach, J. Chem. Phys. 148, 193830 (2018).

[40] A. A. Veldhorst and M. C. C. Ribeiro, Mechanical Heterogeneity in Ionic Liquids, J. Chem. Phys. 148, 193803 (2018).

[41] M. Chen, Z.A.H. Goodwin, G. Feng, and A. A. Kornyshev, On the Temperature Dependence of the Double Layer Capacitance of Ionic Liquids, J. Electroanal. Chem. 819, 347 (2018).

[42] N. Gavish, D. Elad, and A. Yochelis, From Solvent-Free to Dilute Electrolytes: Essential Components for a Continuum Theory, J. Phys. Chem. Lett. 9, 36 (2018).

[43] B. Rotenberg, O. Bernard, and J.-P. Hansen, Underscreening in Ionic Liquids: A First Principles Analysis, J. Phys. Condens. Matter 30, 054005 (2018).

[44] J. G. Kirkwood, Statistical Mechanics of Liquid Solutions, Chem. Rev. 19, 275 (1936).

[45] P. Attard, Asymptotic Analysis of Primitive Model Electrolytes and the Electrical Double Layer, Phys. Rev. E 48, 3604 (1993).

[46] R. Kjellander and D. J. Mitchell, An Exact but Linear and Poisson-Boltzmann-like Theory for Electrolytes and Colloid Dispersions in the Primitive Model, Chem. Phys. Lett. 200, 76 (1992).

[47] R. J. F. L. de Carvalho and R. Evans, The Decay of Correlations in Ionic Fluids, Mol. Phys. 83, 619 (1994).

[48] R. Kjellander, Focus Article: Oscillatory and Long-Range Monotonic Exponential Decays of Electrostatic Interactions in Ionic Liquids and Other Electrolytes: The Significance of Dielectric Permittivity and Renormalized Charges, J. Chem. Phys. 148, 193701 (2018).

[49] A. Ciach, Simple Theory for Oscillatory Charge Profile in Ionic Liquids near a Charged Wall, J. Mol. Liq. 270, 138 (2018).

[50] D. R. MacFarlane, M. Forsyth, E. I. Izgorodina, A. P. Abbott, G. Annat, and K. Fraser, On the Concept of Ionicity in Ionic Liquids, Phys. Chem. Chem. Phys. 11, 4962 (2009).
[51] H. K. Kashyap, H. V. R. Annapureddy, F. O. Raineri, and C. J. Margulis, How Is Charge Transport Different in Ionic Liquids and Electrolyte Solutions? J. Phys. Chem. B 115, 13212 (2011).

[52] K. R. Harris, Can the Transport Properties of Molten Salts and Ionic Liquids Be Used to Determine Ion Association? J. Phys. Chem. B 120, 12135 (2016).

[53] Y. Zhang and E. J. Maginn, Direct Correlation between Ionic Liquid Transport Properties and Ion Pair Lifetimes: A Molecular Dynamics Study, J. Phys. Chem. Lett. 6, 700 (2015).

[54] B. Kirchner, F. Malberg, D. S. Firaha, and O. Hollóczki, Ion Pairing in Ionic Liquids, J. Phys. Condens. Matter 27, 463002 (2015).

[55] I. M. Sokolov, Models of Anomalous Diffusion in Crowded Environments, Soft Matter 8, 9043 (2012).

[56] B. Mandelbrot, How Long Is the Coast of Britain? Statistical Self-Similarity and Fractional Dimension, Science 156, 636 (1967).

[57] The small discrepancy at higher temperatures with more anions than cations being free means the appearance of positively charged clusters of bound ions. Generally, this kind of difference between anions and cations could be expected in view of the propensity of cations to aggregate in quasimicellar structures due to the nearly neutral tails of the cations. However, for the RTILs studied here, the neutral tails of the cations may not be long enough to cause a profound structural heterogeneity. But even if they do, the neighboring anions could bound to and majorly neutralize them. Thus, within the most relevant temperature range, the clusters of bound ions seem to stay neutral.

[58] M. Sha, H. Dong, F. Luo, Z. Tang, G. Zhu, and G. Wu, Dilute or Concentrated Electrolyte Solutions? Insight from Ionic Liquid/Water Electrolytes, J. Phys. Chem. Lett. 6, 3713 (2015).

[59] X.-Y. Wang, R. D. Raharjo, H. J. Lee, Y. Lu, B. D. Freeman, and I. C. Sanchez, Molecular Simulation and Experimental Study of Substituted Polyacetylenes: Fractional Free Volume, Cavity Size Distributions and Diffusion Coefficients, J. Phys. Chem. B 110, 12666 (2006).

[60] W. Beichel, P. Eiden, and I. Krossing, Establishing Consistent van der Waals Volumes of Polyatomic Ions from Crystal Structures, Chem. Phys. Chem. 14, 3221 (2013).

[61] S. Seki, T. Kobayashi, Y. Kobayashi, K. Takei, H. Miyashiro, K. Hayamizu, S. Tsuzuki, T. Mitsugi, and Y. Umebayashi, Effects of Cation and Anion on Physical Properties of Room-Temperature Ionic Liquids, J. Mol. Liq. 152, 9 (2010).

[62] P. J. Stiles and J. B. Hubbard, Electrostriction and Dielectric Friction on Ions Moving through Compressible Polar Solvents, Chem. Phys. Lett. 105, 655 (1984).

[63] N. V. Brilliantov, V. P. Denisov, and P. L. Krapivsky, Generalized Stokes-Einstein-Debye Relation for Charged Brownian Particles in Solution, Physica (Amsterdam) 175A, 293 (1991).

[64] J. R. Zimmerman and W. E. Brittin, Nuclear Magnetic Resonance Studies in Multiple Phase Systems: Lifetime of a Water Molecule in an Adsorbing Phase on Silica Gel, J. Phys. Chem. 61, 1328 (1957). 
[65] B. Halle and H. Wennerström, Interpretation of Magnetic Resonance Data from Water Nuclei in Heterogeneous Systems, J. Chem. Phys. 75, 1928 (1981).

[66] P.-O. Westlund and H. Wennerström, NMR Lineshape for Spin-32 Nuclei in a Heterogeneous System. ChemicalExchange Effects and Intensity Loss of the Central Signal, J. Magn. Reson. (1969) 81, 68 (1989).

[67] A. G. Palmer, J. Williams, and A. McDermott, Nuclear Magnetic Resonance Studies of Biopolymer Dynamics, J. Phys. Chem. 100, 13293 (1996).

[68] E. Berggren and P.-O. Westlund, Theoretical Nuclear Magnetic Resonance-Lineshape Study of a Two-Site Chemical Exchange Model of Sodium Ions $(I=3 / 2)$ in an Intracellular Environment, Biophys. J. 58, 167 (1990).

[69] J. A. Padró, L. Saiz, and E. Guàrdia, Hydrogen Bonding in Liquid Alcohols: A Computer Simulation Study, J. Mol. Struct. 416, 243 (1997).

[70] E. Guàrdia, J. Martí, L. García-Tarrés, and D. Laria, A Molecular Dynamics Simulation Study of Hydrogen Bonding in Aqueous Ionic Solutions, J. Mol. Liq. 117, 63 (2005).

[71] W. Zhao, F. Leroy, B. Heggen, S. Zahn, B. Kirchner, S. Balasubramanian, and F. Müller-Plathe, Are There Stable Ion-Pairs in Room-Temperature Ionic Liquids? Molecular Dynamics Simulations of 1-n-butyl-3-methylimidazolium Hexafluorophosphate, J. Am. Chem. Soc. 131, 15825 (2009).

[72] H. Risken, The Fokker-Planck Equation (Springer-Verlag Berlin, 1984).

[73] R. Metzler and J. Klafter, The Random Walk's Guide to Anomalous Diffusion: A Fractional Dynamics Approach, Phys. Rep. 339, 1 (2000).

[74] B. J. Berne and G. D. Harp, On the Calculation of Time Correlation Functions, in Advances in Chemical Physics, edited by I. Prigogine and S. A. Rice (John Wiley \& Sons, Inc., New York, 1970).

[75] N. Brilliantov and O. Revokatov, Molecular Dynamics of Disordered Media (Publishing House of Moscow State University, Moscow, 1996).

[76] H. Tokuda, K. Hayamizu, K. Ishii, M. A. B. H. Susan, and M. Watanabe, Physicochemical Properties and Structures of Room Temperature Ionic Liquids. 2. Variation of Alkyl Chain Length in Imidazolium Cation, J. Phys. Chem. B 109, 6103 (2005).

[77] C. Rey-Castro and L. F. Vega, Transport Properties of the Ionic Liquid 1-ethyl-3-methylimidazolium Chloride from Equilibrium Molecular Dynamics Simulation. The Effect of Temperature, J. Phys. Chem. B 110, 14426 (2006).

[78] S. M. Chathoth, E. Mamontov, S. Dai, X. Wang, P. F. Fulvio, and D. J. Wesolowski, Fast Diffusion in a Room Temperature Ionic Liquid Confined in Mesoporous Carbon, Europhys. Lett. 97, 66004 (2012).

[79] Y.-L. Wang, M. R. Shimpi, S. Sarman, O. N. Antzutkin, S. Glavatskih, L. Kloo, and A. Laaksonen, Atomistic Insight into Tetraalkylphosphonium Bis(oxalato)borate Ionic Liquid/Water Mixtures. 2. Volumetric and Dynamic Properties, J. Phys. Chem. B 120, 7446 (2016).

[80] With some reservation, such motion resembles the channel diffusion of sodium ions in silicate glasses, although the diffusion of sodium ions in amorphous $\mathrm{Na}_{2} \mathrm{O}-4 \mathrm{SiO}_{2}$ is 2 orders of magnitude faster than that of silicon and oxygen atoms, which allows consideration of the sodium ions diffusing inside a frozen matrix of $\mathrm{SiO}_{2}$; in RTILs, the diffusion coefficients of the free and bound ions are not that much different [81-83].

[81] P. Jund, W. Kob, and R. Jullien, Channel Diffusion of Sodium in a Silicate Glass, Phys. Rev. B 64, 134303 (2001).

[82] A. Meyer, J. Horbach, W. Kob, F. Kargl, and H. Schober, Channel Formation and Intermediate Range Order in Sodium Silicate Melts and Glasses, Phys. Rev. Lett. 93, 027801 (2004).

[83] M. Bauchy and M. Micoulaut, From Pockets to Channels: Density-Controlled Diffusion in Sodium Silicates, Phys. Rev. B 83, 184118 (2011).

[84] J.P. Hansen and I. R. McDonald, Theory of Simple Liquids, 3rd ed. (Academic Press, Philadelphia, 2006).

[85] C. A. Croxton, Liquid State Physics: A Statistical Mechanical Introduction (Cambridge University Press, London, 1973).

[86] M. Kanakubo, K. R. Harris, N. Tsuchihashi, K. Ibuki, and M. Ueno, Temperature and Pressure Dependence of the Electrical Conductivity of 1-butyl-3-methylimidazolium Bis(trifluoromethanesulfonyl)amide, J. Chem. Eng. Data 60, 1495 (2015).

[87] K. R. Harris, Relations between the Fractional StokesEinstein and Nernst-Einstein Equations and Velocity Correlation Coefficients in Ionic Liquids and Molten Salts, J. Phys. Chem. B 114, 9572 (2010).

[88] M. H. Kowsari, S. Alavi, B. Najafi, K. Gholizadeh, E. Dehghanpisheh, and F. Ranjbar, Molecular Dynamics Simulations of the Structure and Transport Properties of Tetra-butylphosphonium Amino Acid Ionic Liquids, Phys. Chem. Chem. Phys. 13, 8826 (2011).

[89] The results in Fig. 7 remind us of the conclusion of Ref. [37] (see also Ref. [90]) stating that "decoupling between diffusion and viscosity observed as the failure of the Stokes-Einstein-Debye relation can be understood on the basis that mobile particles contribute to diffusion, whereas slow particles contribute to structural relaxation." This conclusion is similar in spirit to our findings, but the nature of the effect is different. The effect found in Ref. [37] is mainly associated with large micellarlike clusters of cations that move slowly and have different relaxation times, whereas we investigate the continuous exchange between the bound and free states, which occurs both for cations and anions in a pretty similar fashion.

[90] D. Jeong, D. Kim, M. Y. Choi, H. J. Kim, and Y. Jung, Dynamic Heterogeneity in Room-Temperature Ionic Liquids, in Ionic Liquids: Theory, Properties, New Approaches, edited by A. Kokorin (InTech, Shanghai, 2011), p. 167.

[91] M. Kanakubo, K. R. Harris, N. Tsuchihashi, K. Ibuki, and M. Ueno, Effect of Pressure on Transport Properties of the Ionic Liquid 1-butyl-3-methylimidazolium Hexafluorophosphate, J. Phys. Chem. B 111, 2062 (2007).

[92] A. P. Abbott, Application of Hole Theory to the Viscosity of Ionic and Molecular Liquids, Chem. Phys. Chem. 5, 1242 (2004). 
[93] A. P. Abbott, Model for the Conductivity of Ionic Liquids Based on an Infinite Dilution of Holes, Chem. Phys. Chem. 6, 2502 (2005).

[94] M. Grundmann, The Physics of Semiconductors (Springer, Berlin, 2006).

[95] Y. Y. Gurevich and Y. I. Kharkats, Features of the Thermodynamics of Superionic Conductors, Sov. Phys. Usp. 25, 257 (1982).

[96] M. Salamon, Physics of Superionic Conductors (SpringerVerlag, Berlin, 1979).

[97] K. S. Kim and W.-K. Paik, Effects of Temperature and Pressure on Conductance of Solid Electrolyte, Rubidium Silver Iodide, J. Chem. Eng. Data 20, 356 (1975).

[98] M. L. Knotek and C.H. Seager, The Absence of $a$ Measurable Hall Effect in the Superionic Conductor $\operatorname{RbAg}_{4} \mathrm{I}_{5}$, Solid State Commun. 21, 625 (1977).

[99] C. H. J. Stuhrmann, H. Kreiterling, and K. Funke, Ionic Hall Effect Measured in Rubidium Silver Iodide, Solid State Ionics 154-155, 109 (2002).

[100] H. J. Kroh and B. U. Felderhof, On the Theory of the Hall Effect in Ionic Solutions, Physica (Amsterdam) 153A, 73 (1988).

[101] H. J. Kroh and B. U. Felderhof, The Hall Effect in Dilute Ionic Solutions, Mol. Phys. 70, 119 (1990).

[102] B. Hess, C. Kutzner, D. van der Spoel, and E. Lindahl, GROMACS 4: Algorithms for Highly Efficient, LoadBalanced, and Scalable Molecular Simulation, J. Chem. Theory Comput. 4, 435 (2008).

[103] O. Borodin, Polarizable Force Field Development and Molecular Dynamics Simulations of Ionic Liquids, J. Phys. Chem. B 113, 11463 (2009).

[104] J. Vatamanu, L. Cao, O. Borodin, D. Bedrov, and G. D. Smith, On the Influence of Surface Topography on the Electric Double Layer Structure and Differential Capacitance of Graphite/Ionic Liquid Interfaces, J. Phys. Chem. Lett. 2, 2267 (2011).
[105] P. L. Krapivsky, S. Redner, and E. Ben-Naim, A Kinetic View of Statistical Physics (Cambridge University Press, Cambridge, England, 2010).

[106] Note that in Ref. [105] written mainly for specialists in diffusion kinetics, the dimensional quantities with $D=1$ and $\tau_{0}=1$ have been used, making final estimates less obvious. Since our article is addressed to a wider audience of "end users," we prefer to operate with quantities of explicit and correct dimensionality, although for the qualitative analysis such a "difference" may not be critical.

[107] O. Y. Sliusarenko, V. Y. Gonchar, A. V. Chechkin, I. M. Sokolov, and R. Metzler, Kramers-like Escape Driven by Fractional Gaussian Noise, Phys. Rev. E 81, 041119 (2010).

[108] See Supplemental Material at http://link.aps.org/ supplemental/10.1103/PhysRevX.9.021024 for (1) comparison of results for the temperature-dependent percentage of free ions obtained by three different methods; (2) detailed study of the kinetics of exchange process characterized through survival probabilities for anions in [Bmim][TFSI]; (3) additional analysis of the velocityautocorrelation functions (VACFs) and diffusion coefficients; (4) demonstration of how (exceptionally) well the two state theory developed in the main text can fit the simulated VACFs for anions in [Bmim][TFSI]; (5) analysis of the temperature effect on electric-current autocorrelation function (ECACF) and its Fourier spectrum; (6) analysis of ECACFs and the corresponding values of electrical conductivities obtained by integration of ECAFCs over time; additional information on the results obtained for two other RTILs [Emim][TFSI] and [Bmim] [PF6], including the temperature-dependent (7) percentage of free ions and (8) conductivities; (9) study of the effect of the simulation system size on the ion diffusion coefficient. 